\title{
Evaluation of the Care and Communication Bundle to Improve Palliative Care in Intensive Care Units
}

\author{
Angela Susan Bohon \\ West Virginia University, abohon@mix.wvu.edu
}

Follow this and additional works at: https://researchrepository.wvu.edu/etd

Part of the Critical Care Nursing Commons, and the Other Nursing Commons

\section{Recommended Citation \\ Bohon, Angela Susan, "Evaluation of the Care and Communication Bundle to Improve Palliative Care in Intensive Care Units" (2020). Graduate Theses, Dissertations, and Problem Reports. 7760. \\ https://researchrepository.wvu.edu/etd/7760}

This Problem/Project Report is protected by copyright and/or related rights. It has been brought to you by the The Research Repository @ WVU with permission from the rights-holder(s). You are free to use this Problem/Project Report in any way that is permitted by the copyright and related rights legislation that applies to your use. For other uses you must obtain permission from the rights-holder(s) directly, unless additional rights are indicated by a Creative Commons license in the record and/ or on the work itself. This Problem/Project Report has been accepted for inclusion in WVU Graduate Theses, Dissertations, and Problem Reports collection by an authorized administrator of The Research Repository @ WVU. For more information, please contact researchrepository@mail.wvu.edu. 


\title{
Evaluation of the Care and Communication Bundle to Improve Palliative Care in Intensive Care Units
}

\author{
Angela S. Bohon, MSN, APRN, FNP-C \\ West Virginia University
}

Doctoral Capstone Paper submitted

to the School of Nursing at

West Virginia University

\begin{abstract}
In partial fulfillment of the requirements for the degree of
\end{abstract}
Doctor of Nursing Practice

Lori Constantine, DNP, APRN, FNP-BC, ACHPN, Chair

Anand Pullapilly, MD

Department of Nursing

Morgantown, West Virginia

2020

Keywords: Care and Communication Bundle, Palliative Care, Intensive Care

Copyright 2020: Angela S. Bohon 


\section{Abstract \\ Evaluation of the Care and Communication Bundle to Improve Palliative Care in Intensive Care Units}

\section{Angela S. Bohon}

Review of local data and concerns from nursing staff revealed the need to improve palliative care in a local, community hospital Intensive Care Unit (ICU). This effort was mobilized by implementing the Care and Communication Bundle within evidence-based time frames, into this local, community ICU, using Lewin's Change theory as a guide for implementation. The Care and Communication Bundle is a grouping of nine palliative, quality measures found to improve palliative care in the ICU. A pre/post-educational intervention evaluated if the implementation of the Care and Communication Bundle in the ICU improved palliative care by reviewing the documentation of the nine, palliative, quality measures within selected time frames. The implementation and adoption of the Care and Communication Bundle in a local, community ICU significantly increased four of the nine, palliative, quality measures within those periods. These four measures included palliative leaflet distribution (p-value $<$ 0.01 , social work visitation $(p$-value $=0.02)$, spiritual care support $(p$-value $<0.01)$, and the completion of an interdisciplinary meeting ( $\mathrm{p}$-value $<0.01$ ). The five, palliative, quality measures that did not show significant improvement included documentation of appropriate medical decision maker $(\mathrm{p}$-value $=0.44)$, an advance directive on the chart $(\mathrm{p}$-value $=0.07)$, resuscitation status ( $\mathrm{p}$-value $=0.24)$, regular pain assessment, and optimal pain management. 


\section{Acknowledgements}

I would like to sincerely thank my capstone committee for all of their assistance and support throughout this project. Dr. Lori Constantine, Dr. Anand Pullapilly, and Lisa Simon have been invaluable mentors, educators and clinicians. Their leadership and knowledge guided me through the completion of my capstone. I would also like to thank the Doctor of Nursing faculty of West Virginia University that helped me through the papers and presentations. I would also like to thank Dr. Sandra Cotton for her encouraging words of advice and support throughout this process. I must also acknowledge the staff members of Mon Health Medical Center which supported me throughout the capstone project and never lost faith in me. Attainment of my Doctor in Nursing Practice would not have been possible if it was not for the unconditional support from my friends and family. 


\section{Table of Contents}

$\begin{array}{ll}\text { Introduction } & 7\end{array}$

$\begin{array}{ll}\text { Background } & 7\end{array}$

$\begin{array}{ll}\text { Chronic Disease } & 7\end{array}$

$\begin{array}{ll}\text { Preferences for Care } & 8\end{array}$

Need to Improve ICU Palliative Care 9

$\begin{array}{ll}\text { Impact to West Virginia } & 10\end{array}$

$\begin{array}{lr}\text { Impact at Project Medical Center } & 10\end{array}$

$\begin{array}{ll}\text { Problem Statement } & 11\end{array}$

Purpose of Project $\quad 11$

Significance of Proposed Project 12

$\begin{array}{ll}\text { Literature Review } & 12\end{array}$

$\begin{array}{ll}\text { Methodology } & 13\end{array}$

$\begin{array}{ll}\text { Research Studies } & 13\end{array}$

$\begin{array}{ll}\text { Synthesis } & 17\end{array}$

$\begin{array}{ll}\text { Theoretical Framework } & 17\end{array}$

$\begin{array}{ll}\text { Key Elements } & 17\end{array}$

$\begin{array}{ll}\text { Project } & 18\end{array}$

Description and Design of Evidence-based Project/Intervention Plan 18

$\begin{array}{ll}\text { Patient Population } & 18\end{array}$

$\begin{array}{ll}\text { Sampling Plan } & 19\end{array}$

$\begin{array}{ll}\text { Pre-Intervention Data Collection } & 19\end{array}$

$\begin{array}{ll}\text { Education and Implementation } & 20\end{array}$ 
$\begin{array}{ll}\text { Post Intervention Data Collection } & 21\end{array}$

$\begin{array}{ll}\text { Congruence with Theoretical Framework } & 21\end{array}$

$\begin{array}{ll}\text { Feasibility Analysis } & 22\end{array}$

SWOT Analysis $\quad 22$

$\begin{array}{ll}\text { Resources } & 23\end{array}$

Congruence of the Organizations Strategic Plan to Project 24

Evidence of Key Site Support $\quad 24$

$\begin{array}{ll}\text { Project Objectives } & 25\end{array}$

Evaluation of Objectives $\quad 26$

$\begin{array}{ll}\text { Results } & 27\end{array}$

$\begin{array}{ll}\text { Quality Palliative Measures } & 27\end{array}$

$\begin{array}{ll}\text { Day } 1 & 27\end{array}$

$\begin{array}{ll}\text { Day } 3 & 28\end{array}$

$\begin{array}{ll}\text { Day } 5 & 28\end{array}$

Nurse Survey Results $\quad 29$

$\begin{array}{ll}\text { Facilitators and Barriers } & 29\end{array}$

$\begin{array}{ll}\text { Unintended Consequences } & 30\end{array}$

Discussion and Recommendations 30

How Theory Supported this Project 30

Site Recommendations 31

Application Recommendations 32

$\begin{array}{ll}\text { Conclusions } & 32\end{array}$

Attainment of DNP Essentials 33 
$\begin{array}{ll}\text { References } & 36\end{array}$

$\begin{array}{ll}\text { Tables } & 39\end{array}$

$\begin{array}{ll}\text { Figures } & 46\end{array}$

$\begin{array}{ll}\text { Appendices } & 57\end{array}$ 
Evaluation of the Care and Communication Bundle to Improve Palliative Care in Intensive Care Units

Introduction

Many people die in an ICU. In 2015, 32.4\% of West Virginia (WV) deaths occurred in a WV hospital (West Virginia Vital Statistics, 2015). Palliative care is a crucial part of a patient's hospital stay, improves patient's quality of life, and helps patients and families understand medical treatment choices (Vuong et al., 2019). Most patients spend their time in the ICU without palliative care, results in care that is neither wanted, nor effective. This project describes how an evidence-based, palliative care bundle, known as the Care and Communication Bundle was implemented and partially adopted into a local, community hospital's ICU. Lewin's Change Theory was used to guide the implementation and evaluation of the project.

\section{Background}

\section{Chronic Disease}

Heart disease is the leading cause of death in the Mountain State accounting for 4,819 deaths in WV (Centers for Disease Control [CDC], 2017). Cancer and chronic lower respiratory diseases are the remaining two leading causes of death, accounting for 6,546 West Virginia resident deaths in 2017. Cancer accounts for 4,654 and chronic lower respiratory diseases account for 1,892 deaths in WV (CDC, 2017). There is a high probability that in their lifetime West Virginians will be diagnosed with at least one chronic disease and be admitted to the ICU. Following the current trajectory and expected rise in life expectancy, these patients will occupy an increase amount of critical care beds and their disease states worsen.

\section{Preferences for Care}


In $2015,32.4 \%$ of West Virginians died in a WV hospital, $6.8 \%$ in an out-of-state hospital, $14.2 \%$ in a licensed nursing home, and $29.4 \%$ of deaths occurring at home. Only $9.7 \%$ of WV deaths happened within hospice care (West Virginia Vital Statistics, 2015). Most West Virginians wish to die at home in the presence of their family. West Virginia Center for End-ofLife Care provides coordination, education, and resources so that West Virginians can die in the setting of their choice.

Overall, adult patients who are admitted to an ICU have an average mortality rate of $10 \%$ to $29 \%$ (West Virginia Vital Statistics, 2015). Patients with multiple chronic diseases are prone to admission to the ICU. When patients are hospitalized, they are often faced with information regarding their own mortality. Moreover, when patients are critically ill, their families are faced with making difficult decisions regarding the patients' future plans of care, which can be overwhelming. When delivering crucial information to a patient and family, it is a critical time to implement palliative care, as it addresses all aspects of life.

Palliative care involves psychological, emotional, and spiritual wellness, as well as pain and symptom management, often delivered alongside curative therapies. With palliative care, individuals do not have to give up treatments that might cure a serious illness. Focusing on the patients' and family's goals of care is extremely important (Wessman et al., 2016). For appropriate care to be delivered in future hospital stays, clear documentation of goals of care is crucial.

Patients and families can express their goals of care in many ways. Often these goals are converted to legal documents such as advance directives, medical power of attorney (MPOA) or healthcare surrogate (HCS) forms, and medical orders such as do-not-resuscitate (DNR) cards, and physician scope of treatment (POST) forms. These forms reflect ongoing advance care 
planning conversations and are best achieved through involvement of the interdisciplinary team. Appropriate documentation of the patient's goals of care is important in providing quality palliative care. The Care and Communication Bundle promotes timely documentation of nine palliative care measures by recommended, specific time frames. Documentation of the following must occur by day one, day three, and day five:

- Day one: (1) appropriate decision maker, (2) advance directive, (3) resuscitation status, (4) distribution of a palliative care, informational leaflet (Figure 1), (5) regular pain assessments, and (6) optimal pain management.

- Day three: (7) social work visit, (8) pastoral care or spiritual support offering

- Day five: (9) interdisciplinary family meeting.

\section{Need to Improve ICU Palliative Care}

In 1996, a ground-breaking study revealed the extent of poor quality of palliative care in ICUs. This randomized control trial, Study to Understand Prognoses and Preferences for Outcomes and Risks of Treatments (SUPPORT) (1996), focused on improving interdisciplinary communications including physicians, nurses, patients, and families. This study also attempted to improve pain assessment and management, increase the frequency of interdisciplinary, family meetings, and finally, identify and communicate patients' prognoses, goals of care, and at times, end-of-life-care options. This seminal study supported the crucial need for future research regarding how to improve palliative care in the ICU.

Conversations surrounding resuscitation status, appointment of medical decision makers, advanced directives, and palliative care remain stigmatized and infrequent (ALMouaalamy et al., 2018). Physicians, nurse practitioners, physician assistants, and nurses have reported a lack of education on how to facilitate these conversations. Subsequently, the lack of such discussions 
early in the disease process, or worse, upon admission or transfer to an ICU often results in palliative care occurring at a very late stage in the disease process. Research shows that early implementation of palliative care results in beneficial outcomes for patients (Nelson et al., 2006). Although an ICU admission can be late in a patient's disease trajectory, early intervention of the Care and Communication Bundle is crucial for the ICU patient.

Impact to West Virginia. West Virginia lies almost completely in the Appalachian Mountains in the Mid-Atlantic region of the United States (US). WV is bordered by Maryland, Virginia, Pennsylvania, Ohio, and Kentucky. In 2010, the population per square mile was 77.1 persons. Almost $18 \%$ of West Virginians live in poverty, and nearly $14 \%$ have not graduated from high school. A total of nearly $8 \%$ of West Virginians live without insurance and $14.1 \%$ live with a disability (U.S. Census Bureau, 2020). In 2018, 39.5\% of WV adults were obese, 20.3\% of children of ages $10-17$ were obese, and $25.2 \%$ of West Virginians smoked cigarettes. Chronic diseases are prevalent in West Virginians; $16.2 \%$ of adults have diabetes, and $43.5 \%$ of adults have hypertension (U.S. Census Bureau, 2020). West Virginia ranked 46th in 2019 as one of the unhealthiest states in the United States of America (Findings State Rankings; 2019 Senior Report, 2020). West Virginians experience barriers in receiving primary healthcare due to lack of resources, living in rural areas, and lack of insurance. WV is ranked second to last as the state with the lowest average household income. The average household income for WV in 2019 was $\$ 66,563.23$ (Average Income by State, Median, Top \& Percentiles, 2019). Due to patient's lack of primary healthcare, patients tend to need higher level of care, requiring ICU hospitalization.

Impact at project medical center. In 2010, there were 96,189 persons living in Monongalia County, WV. Approximately 13\% of the population of Monongalia County was persons 65 years or older, whereas the percentage of persons 65 or older in the state of WV is $19.9 \%$ (U.S. Census 
Bureau, 2020). There is one major city in Monongalia County, which is Morgantown. Located within Morgantown are two hospitals, one is a 189-bed community hospital and the other is a 652-bed academic medical center. The community hospital lacks a palliative care team at the time of this project's implementation. Data and information supporting the need of an inpatient palliative care consult team is currently unknown. The implementation and completion of this project is crucial to determining the need for palliative care at this community hospital.

\section{Problem Statement}

Although there are multiple opportunities for providers to address resuscitation status, advance directives, and other palliative care measures, these opportunities are often not explored. Multiple patients are being admitted to the ICU at the local community hospital without a resuscitation status, advance directive on file, or medical decision maker (one or more of the nine quality measures). Often, these aspects aren’t addressed multiple days after admission to ICU. In the pre-intervention phase, it was found that $33 \%$ of patients on day one in the ICU lacked documentation of resuscitation status. Palliative care has been recognized as a priority in ICU patient care, and is not been provided consistently.

\section{Purpose of Project}

The purpose of the quality improvement project is to implement the Care and Communication Bundle in patients who are admitted or transferred to the ICU to prevent delayed palliative care, and to improve the quality of palliative care. The goal of this project is to increase timely documentation of the nine quality measures of palliative care (Appendix A), which will positively impact palliative care received at this local community hospital.

\section{Significance of Proposed Project}


This project will have a significant impact for the patients, their families, and the healthcare providers at the local, community ICU. A major concern of the facility where the project occurred was the lack of documentation of resuscitation status and advanced directives on file by day one of an ICU admission. Approximately $50 \%$ of patients at the project implementation site did not have a documented resuscitation status in the electronic medical record (EMR). Moreover, less than half of those patients had an advanced directive on file. The facility was right to be concerned. These are two of six crucial elements of quality palliative care that occur on day one of an ICU stay. Without appropriate documentation, a delay in implementing palliative care in the ICU occurs. Therefore, the implementation of the Care and Communication Bundle is expected to improve palliative care for the patients and families in the ICU at this community hospital.

\section{Literature Review}

The purpose of this literature review is to determine which interventions have been effective in improving palliative care in the ICU setting. This literature review yielded studies which implemented the Care and Communication Bundle as well as studies that solely implemented an interdisciplinary palliative care team. The Care and Communication Bundle focused on nine palliative, quality measures; therefore, emphasis was placed on literature regarding end of life care bundles. The literature reviewed supports implementation of bundles such as the Care and Communication Bundle, as well as implementation of interdisciplinary teams to increase palliative care measures within the ICUs (Constantine, 2016; Gade et al., 2008; Vuong et al., 2019; Wessman et al., 2016).

\section{Methodology}


The databases utilized for this literature search included CINAHL, and MEDline. The original search included the key words "integrating palliative care" which resulted in the return of 102 publications. To narrow the search, a date range was added to only include publications from 2006 - 2019, which then yielded 91 publications. Many successful and crucial studies regarding the implementation of the Care and Communication Bundle were beyond the usual 10year timeframe, therefore this literature search was expanded to 13 years. Limitations were added which included peer reviewed, English language, and academic journals; 76 publications were yielded. The titles of 76 publications were reviewed for relevance. Keyword "ICU" was then added to the search which yielded 7 solo hits. The Center to Advance Palliative Care (CAPC) was used to search Integrating Palliative Care Practices in ICU, which yielded 41 articles. The snowball technique was used from relevant articles which yielded 37 articles. Using these articles, inclusion and exclusion criteria were applied. Inclusion criteria included ICU stay, implementing Care and Communication, and palliative care. Exclusion criteria included pediatric ICU, outpatient hospice, and pregnant persons. These methods led to a total of 5 relevant articles for review: Constantine (2016), Gade et al. (2008), Nelson et al. (2006), Vuong et al. (2019), and Wessman et al. (2016).

\section{Research Studies}

There were two types of studies that were reviewed, four of which were preintervention/postintervention studies: Constantine (2016), Nelson et al. (2006), Vuong et al. (2019), and Wessman et al. (2016), and one being a randomized, control study: Gade et al. (2008). Three of the five studies studied the implementation of the Care and Communication Bundle on the nine palliative care quality measures within the ICU: Constantine (2016), Nelson et al. (2006), and Vuong et al. (2019), The remaining two studies evaluated the implementation 
of multidisciplinary and interdisciplinary palliative care teams on palliative care measures, but not specific to the nine quality care measures within the Care and Communication Bundle (Gade et al. (2008), and Wessman et al. (2016). All studies were implemented solely within an ICU except for the study completed by Gade et al. (2008). Three studies provided strong evidence of support for increased palliative care measures: Gade et al. (2008), Vuong et al. (2019), and Wessman et al. (2016). Two studies demonstrated partial support of increased palliative care measures, based on intervention, and needed to be studied further: Constantine (2016), Nelson et al. (2006).

Wessman et al. (2016), completed a preintervention/postintervention study that evaluated the implementation of set guidelines specific to end of life care, over a two-year period. This study was conducted at an academic tertiary surgical trauma 24-bed surgical burn trauma ICU and then was expanded to a 36-bed surgical burn trauma ICU. Wessman et al. (2016), supported that educational programs related to palliative care increase the quality of palliative care received in the ICU. Wessman et al. (2016), studied a multidisciplinary-based training program for improved end of life care. Post implementation of guidelines, results supported there was an improvement in education for family, improved family communication and support, improved order sets for end of life care, and overall improved ICU resources available to support end of life care in surgical burn trauma center ICU population.

Vuong et al. (2019), completed a pilot study which included the implementation of the Care and Communication Bundle tool within a 24-bed adult MICU at the University of Florida Health Hospital. The University of Florida Health Hospital is a level 1 trauma, academic, tertiary care center located in north central Florida. A multidisciplinary committee was convened to implement the Care and Communication Bundle in the MICU, which consisted of a champion 
resident physician and a doctorate of nurse practitioner/registered nurse (Vuong et al., 2019). The study of the implementation of the Care and Communication Bundle completed by Vuong et al. (2019), supported that the Care and Communication Bundle was consistent with improving the nine high quality measures of palliative care. Results of this pilot study demonstrated the average length of stay in the ICU was decreased, the number of discharges directly to hospice increased, and an increase in bedside palliative care from providers within the MICU (Vuong et al., 2019). Overall, the implementation of the Care and Communication Bundle at this facility increased the number of goals of care conversations, basic symptom management, discussion of advance directives, and code status (Vuong et al., 2019). This study is now being implemented hospitalwide, with results pending.

Gade et al. (2008), conducted a multicenter, randomized, controlled trial to evaluate the effect of integrating an interdisciplinary palliative care team on overall patient care experience, patient outcomes, and healthcare costs. This study was completed in three hospital settings in Denver, Portland, and San Francisco from June 2002 to December 2003, which consisted of five hundred and seventeen patients with life-limiting illnesses. This study did not include implementation of a bundle; this study implemented an interdisciplinary palliative care service (Gade et al., 2008). Results of this study demonstrated increased palliative care satisfaction in regards to patient's care experience and provider's communication, decreased ICU admissions, and reduced total health care costs (Gade et al., 2008). However, this study was not completed solely in the ICU, but overall this study supports the need of an interdisciplinary palliative care service within the hospital setting to increase palliative care outcomes.

Constantine (2016), implemented a nurse led educational intervention on palliative care using the Care and Communication Bundle program which also had similar positive results. This 
study was completed in a 12-bed surgical ICU in a rural mid-Atlantic region of the United States. In the nurse led educational intervention completed by Constantine (2016), results showed documentation of an appropriate decision maker, advance directives, resuscitation status by day 1, and social work visitation by day 3 increased, documentation of offering a spiritual care visit by day 3 and an interdisciplinary family meeting by day 5 did not increase. Documentation of an appropriate decision maker, advance directives, resuscitation status, and social work contact improved significantly after the implementation of the Care and Communication Bundle (Constantine, 2016). Furthermore, the nurse led implementation of the Care and Communication Bundle was partially successful in improving the documentation of the nine quality measures of palliative care.

Nelson et al. (2006), used an interdisciplinary iterative process to create and evaluate a prototype "bundle" within the ICU. The "bundle" that was used by Nelson et al. (2006), focused on nine domains, which is now also known as the Care and Communication Bundle. Nelson et al. (2006), studied the implementation of this bundle, which included timely documentation of the 10 quality measures at ICU day one, ICU day three, and ICU day five. Medical decision maker, advance directive, resuscitation status, family information leaflet, regular pain assessment, and optimal pain management were the 6 domains evaluated at ICU day one. Social work support and spiritual support were the two domains evaluated at ICU day three. Interdisciplinary family meeting was the final domain evaluated at ICU day five (Nelson et al., 2006). This pilot study was completed at a university affiliated tertiary care hospital and two mixed medical-surgical ICU at separate community hospitals. In the study completed by Nelson et al. (2006), specifications and definitions were used for selected measures in the palliative care bundle, which was considered a limitation. Overall, the conclusion of this study reported that 
further investigation was needed to confirm associations between the implementation of the bundle and outcomes related to patients, and families (Nelson et al., 2006).

\section{Synthesis}

In conclusion, a systematic literature search yielded five articles which consisted of multiple types of studies, including: preintervention/postintervention study, pilot study, multicenter, randomized, controlled trial, nurse led educational intervention study, and interdisciplinary iterative process study. Out of the five publications found, three of the publications suggests that implementation of the Care and Communication Bundle improves at least one of the nine quality measures of palliative care. Overall, publications Constantine (2016), Gade et al. (2008), Vuong et al. (2019), and Wessman et al. (2016) supported the timely improvement of at least one quality care measures with the implementation of the Care and Communication Bundle.

\section{Theoretical Framework}

Adoption of the Care and Communication Bundle in the ICU may pose difficulty due to varying attitudes among healthcare providers about the timing of palliative care and their flexibility with implementing change. Lewin's Change Theory was selected to guide this project's implementation. This theory was chosen due to its ability to simply guide the adoption of the change process and secure that the change remains permanent through his three-stage model of change (Wojciechowski et al., 2016).

\section{Key Elements}

Lewin created a three-stage change model which is known as the "unfreeze - change refreeze" mode (Wojciechowski et al., 2016). The first stage of Lewin's change model is the unfreeze stage. In this stage the current problem must be understood. Education of staff to the 
current problem, the rationale for change, and the way change will occur is crucial for a successful change project. The second stage of Lewin's change model is referred to as the change stage. Change is implemented at this stage, and a new equilibrium is created. Communication, teamwork, role modeling, and training are important to ensure change. The third and final stage of Lewin's change model is referred to as refreeze. This stage ensures that the change becomes permanent including stabilization of change to prevent regression (Wojciechowski et al., 2016). Lewin's Change Theory creates successful change by allowing a preparation of change, implementation of change, and ensuring the change remains permanent.

\section{Project}

\section{Description and Design of Evidence-based Project/Intervention Plan}

The Care and Communication Bundle was first implemented as a performance improvement initiative to improve quality palliative care in the ICU (Nelson et al., 2006). The Care and Communication Bundle's nine palliative, quality measures are "bundled" together, which makes this tool unique. Care and Communication Bundle recommends completion of key quality measures by day one, three, and five of a patient's ICU stay (Appendix A). For this local community hospital's ICU, a pre/post design to determine the effectiveness of the implementation of the Care and Communication Bundle was utilized. The intervention required collaboration of multidisciplinary team members such as physicians, nurse practitioners, registered nurses, social workers, and chaplains.

\section{Patient population.}

This quality improvement project took place in the ICU at the local community hospital in Northern West Virginia, which consists of a total of 16 ICU beds. Types of patients admitted to this unit include vascular, general surgery, general medicine, pulmonary, metabolic diseases, 
renal disease, and cardiac thoracic surgery. The maximum nurse to patient ratio is one to two. The specific target population for this change project included all adults that were admitted or transferred to ICU between December 30, 2019 and February 26, 2020. This did not include adults that were pregnant or younger than 18 years of age.

\section{Sampling plan.}

Prior to data collection a power analysis was used to calculate the minimum sample size required to determine an effect of the given sample size. Power analysis of $80 \%$ was targeted. It was determined that sample sizes of 30 in the pre and post op intervention data collection periods were needed to obtain 0.80 . Most researchers consider 0.80 the standard for adequacy. These requirements adhere to the minimum sample size required by Joint Commission to determine an effect of a given sample size. It can be reasonably concluded that there was a significant difference in pre-intervention data and post-intervention data due to intervention and not by chance when there is a power of 0.80 .

A convenience sample of 30 patients that met inclusion criteria was audited before intervention via the EMR. A second convenience sample of 30 patients that met inclusion criteria were audited post intervention. Pre-intervention data collection included chart reviews from a three-month period of September 1, 2019 through December 1, 2019 prior to the intervention. Implementation of the Care and Communication Bundle occurred between December 30, 2019 and February 26, 2020. Post-intervention data collection occurred from February 27, 2020 and March 15, 2020 for the dates of implementation.

Pre-Intervention data collection. All adult ICU patients that were admitted or transferred to ICU from September 1, 2019 to December 1, 2019 were included in data collection. These patients were then screened for presence of inclusion criteria; being ICU status, 
age greater than 18 years of age, and non-pregnant (Appendix B). If the patient met inclusion criteria, their chart was then audited using a chart abstraction tool (Appendix C) that was also used to obtain information regarding documentation timeframes of the nine quality measures of the Care and Communication Bundle.

Education and implementation. The multiple interventions to promote the adoption of the Care and Communication Bundle in the 16 bed ICU at the local, community hospital occurred between December 30, 2019 and February 26, 2020 and included the following:

1. Nursing education regarding the role in the success of the project, and the potential benefits for the patients and families by nursing staff meetings and individual educational sessions.

2. Three posters, (Figure 2), were placed in highly utilized locations within the ICU to act as a reminder of the nine processes in the Care and Communication Bundle.

3. Pocket cards, (Figure 3), were handed out to the staff members of the ICU, which included nurses, case managers, Advance Practice Provider (APP), physicians, care coordinators, and the nursing director. Pockets cards were also displayed at each nursing station computer as well as computer on wheels as another reminder of the nine processes and time frames in the Care and Communication Bundle.

4. Daily rounding with nursing and physician leadership was utilized to address concerns and questions.

5. Nursing staff also had access to the Care and Communication Bundle checklist, (Appendix A), which detailed the key nine processes and timeframes in each patient chart. 
Post-Intervention data collection. Implementation of the Care and Communication Bundle occurred between December 30, 2019 and February 26, 2020. Post-intervention data collection occurred from February 27, 2020 to March 15, 2020 for the dates of implementation. This quality performance project's primary aim was to assess if the implementation of the Care and Communication Bundle improved palliative care (the nine quality measures) within specific timeframes. The nursing staff in the ICU was instructed to use the Care and Communication Bundle checklist in each chart to determine what quality measures needed addressed with the APP or physician on morning rounds.

\section{Congruence with Theoretical Framework}

Lewin's Change Theory Model acts as a guide for stakeholders to accept and adopt the Care and Communication Bundle into their everyday practice for each patient in the ICU. During the unfreeze stage, education was delivered to staff members including nurses, physicians, APPs regarding why the change is needed. The Care and Communication Bundle checklist was discussed and explained at one staff meeting and individual educational sessions over three weeks (Figure 4). Each of the nine quality measures were discussed in detail during these educational sessions. Finally, questions were answered and a staff educational survey was completed to determine the staff's level of comfort with the Care and Communication Bundle's planned implementation.

During the second stage, which is the change stage, the Care and Communication Bundle checklist was inserted into the front of each paper chart, and a copy was placed in the physician's office. Pocket cards with the nine quality measures and timeframes were distributed to each nurse and were applied to each computer screen at both the nurse's station and the mobile computers. A poster explaining the Care and Communication Bundle along with the nine quality 
measures also was posted in the family meeting conference located in the ICU, and on both ends of the ICU.

The refreeze stage or the final stage of the project was completed with the goal of demonstrating an increased documentation of the nine quality measures of the Care and Communication Bundle. The refreeze of this project is focused on sustaining the Care and Communication Bundle as a standard of practice in the ICU at this local, community hospital. Feedback was later provided to staff members regarding the documentation of the nine quality measures in the Bundle. Staff recommendations were obtained regarding the implementation of the Care and Communication bundle to help predict future sustainability.

\section{Feasibility Analysis}

Without management from a designated team, the implementation of the Care and Communication Bundle is not viable. The implementation of the Care and Communication Bundle at the local community hospital without a designated palliative care team did not yield successful results. With the management of a team, such as a palliative care team the implementation of the Care and Communication Bundle for the local community hospital would be more financially feasible. Under the management of a team designated to palliative care, it is projected that there would be a more definite increase in the nine quality measures, and a decrease in hospital stays. Therefore, the implementation of the Care and Communication Bundle would be feasible as there is a team designated for palliative care, and would not accrue the costs as such for this DNP project, making the Care and Communication Bundle feasible.

\section{SWOT analysis.}

A SWOT analysis was performed in order to analyze the strengths, weaknesses, opportunities, and threats that would be associated with implementing the Care and 
Communication Bundle. The strengths of this project included: teamwork between all staff employed at the local, community hospital, the ability to implement "bundles" through policy, the adaptive physicians that are willing to help implement change. The weaknesses of this project included: resistance to change by bedside nurses, the lack of designated palliative care team, and the lack of interdisciplinary rounds in the ICU. Opportunities that were associated with project included: improved recognition of patients without appropriate documentation of the nine quality measures, and improvement of documentation of four of the nine quality measures within the ICU. The threats that were associated with this project included: the local community hospital consisting of lower acuity of patients, and the lack of specialized services that are offered.

Overall, the project had more strengths than or weaknesses, as well as more opportunities than threats. Therefore, it was decided to implement the Care and Communication Bundle at the community hospital.

\section{Resources.}

The overall project total costs were $\$ 26,413.74$. This total included funding for personal salaries of $\$ 26,118$, (Table 1). However, these costs were deferred since these fees were integrated into existing job responsibilities of all persons involved. There were no additional costs associated with personal salaries. Non-human costs totaled $\$ 295.74$ (Table 2). Supplies utilized for implementation of this project were provided by the investigators.

This project did not directly incur costs to the ICU, however additional time to complete tasks such as documentation were required from staff members, specifically nursing staff. It was expected that the nursing staff would consult the Care and Communication Bundle and document which quality measures had been completed, then address the quality measures that had not been completed. It was also expected that there would be an increase of consultations to social 
services, and to spiritual support. Interdisciplinary family meetings were also expected to increase.

\section{Congruence of the Organization's Strategic Plan to Project}

The lack of a palliative care consult team is an unfortunate reality at the local, community ICU. The strategic plan of this community hospital states they are focused on providing the best, most effective and affordable care to our patients (Mission, Vision \& Values | Mon Health Medical Center, 2019). Implementation of the Care and Communication Bundle is in line with this mission. The implementation of the Care and Communication Bundle focused on providing the most up to date and effective care to all patients in the ICU. The implementation of this project also encouraged nursing staff to provide timely care to patient, which lead to the most effective care to be given.

\section{Evidence of Key Site Support}

A letter of support was obtained from the community hospital prior to project implementation (Figure 5). The project was discussed with the Chief Medical Officer, Dr. Nelcamp, Mon Health Medical Center IRB facilitator, Lisa Simon, and Chief Nursing Executive and VP of Patient Care Services, Daphne Scordato. Management and leadership at Mon Health Medical Center supported the implementation of the Care and Communication Bundle in the 16bed ICU. This project was approved by the hospital, and the protocol was submitted to the Institutional Review Board (IRB). West Virginia University (WVU) IRB determined that this protocol did not meet the definition of human subject's research and was able to proceed with the project on December 3, 2019, supported by (Figure 6).

Once it was determined to proceed with this quality measure project, Administration was contacted prior to initiation of the study. A written copy of the results of this study will be 
provided to the Director of Nursing Administration as well as with the staff members involved within the study.

\section{Project Objectives}

The primary aim of this project was to determine if the adoption of the Care and Communication Bundle impacted palliative care processes for patients admitted to the ICU. In order to ascertain if this aim was met, several objectives were evaluated. Since there are nine quality palliative measures in the Care and Communication Bundle that are documented in the EMR at specific time frames, the specific objectives that were evaluated mirrored these quality measures and their implementation. The nine objectives are:

By Day 1 in ICU:

1. The Care and Communication Bundle will increase documentation of an appropriate decision maker on the EMR.

2. The Care and Communication Bundle will increase the documentation of advance directives in the EMR.

3. The use of the Care and Communication Bundle will increase the documentation of resuscitation status in the EMR.

4. The use of the Care and Communication Bundle will increase the documentation of the distribution of a palliative care informational leaflet.

5. The use of the Care and Communication Bundle will increase regular documentation a pain assessment in the EMR.

6. The use of the Care and Communication Bundle will increase the frequency of optimal pain management that is documented in the EMR.

By Day 3 in the ICU: 
7. The use of the Care and Communication Bundle will increase documentation of social work consult in the EMR.

8. The use of the Care and Communication Bundle will increase documentation of spiritual support consult offered in the EMR.

By Day 5 in the ICU:

9. The use of the Care and Communication Bundle will increase the documentation of interdisciplinary family meetings that have occurred in the EMR.

\section{Evaluation of Objectives}

The nine quality measures were evaluated individually. The percentage of patients with the documentation of an appropriate decision maker, advance directives, resuscitation status, distribution of information leaflet to patients and/or families in the EMR by day one was evaluated using Chi Square. The percentage of four-hour interval documentation of pain assessment and four-hour interval documentation in which pain rating is less than five on a numeric pain scale, for patients in the EMR by day one was evaluated an independent t-test. The percentage of patients or families who had documentation of contact with a member of the care management (social work) team or the offering of spiritual support consult in the EMR by day three was evaluated using Chi Square. Finally, the percentage of patients who had an interdisciplinary family meeting documented in the EMR by day five was evaluated using Chi Square.

Additionally, staff nurse evaluation was obtained to learn firsthand the experience of implementing the Care and Communication Bundle in the ICU. Nurses completed a survey at the end of the implementation using yes/no questionnaire (Appendix D) which evaluated the percentage of staff nurses that found the provided educational sessions helpful in the 
implementation and understanding the significance the Care and Communication Bundle. Additionally, the nurses were asked about the ease of use and perceived effectiveness of the Care and Communication Bundle. Finally, nurses were asked about the feasibility and benefit of whether an organization-wide order set to standardize the implementation of the Care and Communication Bundle.

\section{Results}

\section{Quality Palliative Measures}

The Care and Communication Bundles' quality measures were reviewed as the percentage of the actual times the nine quality measures were documented within the specific time frame required. These time frames were as follows: by day one (identified as the day after ICU admission), identification of an appropriate decision maker, advance directive status, code status, and documentation of patient or family receiving information leaflet, by ICU day three, care management consult and spiritual support offered, and at ICU day five, interdisciplinary family meeting.

Day 1. Percentages and frequencies of the quality measures for both pre-intervention and post-intervention for day one is listed in (Table 3). Evaluation of these quality measures were completed using Chi Square. The MPOA or HCS were documented in the EMR 15 times (50\%) in the pre-intervention phase and 18 times $(60 \%)$ in the post-intervention phase. There was not a significant difference $(p=0.44)$. The presence of an advance directive in the paper chart or in the EMR was documented 10 times (33\%) in the pre-intervention phase, and 17 times $(56 \%)$ in the post-intervention phase, which did not show a significant difference $(p=0.07)$. Resuscitation status was documented 20 times $(67 \%)$ in the pre-intervention phase and 24 times $(80 \%)$ in the post-intervention phase. There was not a significant difference $(p=0.24)$. Lastly, distribution of 
the informative patient leaflet was documented zero times in the pre-intervention phase and 23 times $(76 \%)$ in the post-intervention phase.

Pain assessment as well as optimal pain management was documented by day one in both pre-intervention and post-intervention time periods at 100\%. Regular pain assessment and optimal pain management were both documented at $100 \%$ pre-intervention and postintervention, therefore there is no significant change noted, but warrants further investigation.

Day 3. Percentages and frequencies of the quality measures for both pre-intervention and post-intervention are listed in (Table 4). Evaluation of these two quality measures were completed by using Chi Square. Care management consults were documented 14 times (46\%) in the pre-intervention phase and 23 times $(76 \%)$ in the post-intervention phase, which was a significant difference $(\mathrm{p}=0.02)$. Documentation of spiritual support being offered was completed 10 times (33\%) in the pre-intervention phase and 24 times $(80 \%)$ in the postintervention phase, which was a significant difference $(\mathrm{p}<0.01)$.

Day 5. Percentages and frequencies of the quality measure for the pre-intervention phase and post-intervention phase for quality measure nine is listed in (Table 5). Interdisciplinary meetings were documented 7 times $(24 \%)$ in the pre-intervention phase and 18 times $(60 \%)$ in the postintervention phase, which was a significant difference $(\mathrm{p}<0.01)$. Chi square was used to evaluate for a significant difference between the number of documented interdisciplinary family meetings in the pre-intervention phase and in the post-intervention phase. In conclusion, the aim of this study was to increase the timely documentation of the nine quality palliative measures of the Care and Communication Bundle. The Care and Communication Bundle was successful in significantly increasing the documentation of four out of the nine quality measures.

\section{Nurse Survey Results}


The ICU staff nurses completed a post-intervention survey regarding the implementation of the Care and Communication Bundle in the ICU, which consisted of 25 nurses (Table 6). Upon completion of the survey $84 \%$ of nurses found that provided educational sessions regarding the implementation of the Care and Communication Bundle were helpful, whereas $16 \%$ did not. The educational sessions on educating the importance of implementing the Care and Communication Bundle was found to be helpful by $100 \%$ of the nurses. Twenty-two nurses ( $88 \%)$ documented that they found the Care and Communication Bundle easy to use, and 12\% did not. However, only $76 \%$ of nurses believed the Care and Communication Bundle was effective in the ICU, whereas 24\% did not believe the Care and Communication Bundle was effective. Lastly, 100\% of nurses believed that if the Care and Communication Bundle were an order set, the hospital house wide would benefit from the outcomes on the implementation of the Care and Communication Bundle.

\section{Facilitators and Barriers}

Facilitators that made the nine, quality palliative objective achievable included the support of key stakeholders. Stakeholder support was crucial for the successful implementation of this project to be successful. Key stakeholders for this quality improvement project were physicians, nurses (in both bedside and administrative roles), social workers, pastoral care, and the patients and families.

Key barriers that impacted the nine, quality palliative objective achievable included the change in the nursing staff and the significant increase in case management consults during the implementation of the Care and Communication Bundle. During the implementation phase many nurses returned to the ICU from leave, therefore had not been educated on the Care and Communication Bundle and did not know their role in the project. Many non-ICU nurses were 
floated to the ICU to help with coverage. Again, these nurses were not educated on their role in the implementation of the Care and Communication Bundle. Also, there was a significant increase in case management consults during the implementation of the Care and Communication Bundle probably impacted by the timing of the care manager visit. Since care management did not attend interdisciplinary team rounds in the morning, they did not have the opportunity to speak with the patient as part of the interdisciplinary team, and later were often denied entry to the patient room for various reasons.

\section{Unintended Consequences}

During the implementation of the Care and Communication Bundle, the administration of the local community hospital noticed the lack of palliative care throughout not only the ICU but the oncology unit as well. Results of this project supported the need for a palliative care at this local community hospital. The local community hospital has started to the process of a Palliative Care Team, which already consists of a physician and a nurse. They are looking to grow their palliative care team, and to address the nine quality measures that make up the Care and Communication Bundle.

\section{Discussion and Recommendations}

\section{Discussion}

This DNP project aimed to detect lack of documentation, ancillary involvement, and pain management in critical care patients. Resuscitation status, appointment of medical decision makers, advanced directives, are often poorly explored and documented in healthcare (ALMouaalamy et al., 2018). Literature that supports and recommends that the Care and Communication Bundle leading to an improvement of appropriate documentation, ancillary involvement, and overall patient outcomes ALMouaalamy et al., 2018). Current research and 
technology are attempting to increase how we can adopt implementation of the nine quality measures within the Care and Communication Bundle, however it is limited. While this project was partially successful, the implementation of the Care and Communication Bundle identified gaps in timely documentation of all nine quality, palliative measures.

Results supported four of nine quality, palliative measures significantly improved after the implementation of the Care and Communication Bundle which included palliative leaflet distribution ( $p$-value $<0.01)$, social work visitation $(p$-value $=0.02)$, spiritual care support $(p$ value $<0.01)$, and the completion of an interdisciplinary meeting ( $p$-value $<0.01)$. The quality measures that did not show significant improvement included documentation of appropriate medical decision maker $(\mathrm{p}$-value $=0.44$, and documentation of an advance directive on the chart $(p$-value $=0.07)$ documentation of resuscitation status $(p$-value $=0.24)$

It should be noted that regular pain assessment and optimal pain management were documented at $100 \%$ pre and post intervention, which warranted further investigation. Through informal conversations with hospital staff, lack of pain assessment tools was identified. Current pain assessment tool for all patients, including intubated patients, within the ICU is the numeric pain scale. There are current interventions underway to integrate the Critical Care Pain Observation Tool (CPOT) to ensure patients are assessed appropriately for pain. Therefore, the accuracy of pain assessment and optimal pain management in this study is questionable. Further investigation, and evaluation of regular pain assessment and optimal pain management is recommended.

\section{How Theory Supported this Project}

Lewin's change theory was the most appropriate for the implementation of the Care and Communication Bundle. While starting this project, all staff members had to "unfreeze", which 
consists of allowing change to their set daily routine and practice. Once education took place it was time for the "change" to occur. This is the time that staff members were adapting to change, and how to integrate the Care and Communication Bundle into their new routine. The final step the not all staff members achieved was the "refreeze" step. The majority of staff members experiences little to no difficult into adding the Care and Communication Bundle to their daily routine and was able to "refreeze" easily. The few remaining staff members were stuck in "change", as they were not able to adapt as quickly as others. The staff members and that didn't make it to "refreeze" consisted of part time, and on call employers that had minimal practice time in the ICU.

\section{Site Recommendations}

During both the pre-intervention phase and the post-intervention phase, resuscitation status was documented in the history and physical or was documented in a progress note but was not in the EMR as an active order. Also, during the pre-intervention phase and the postintervention phase advance directives were not always scanned the day of admission, or the day of completion, therefore was not available for staff to determine the exact date it was completed. It is recommended that all admitting physicians or APPs put in an active resuscitation status upon admission to the hospital and be completed upon arrival to the unit in which the patient is admitted. I would recommended that advanced directives are scanned on the first day of ICU admission, or as soon as they become available. I would also recommend integrating additional pain assessment tools within the ICU such as the CPOT and/or the Behavioral Pain Scale (BPS), as well educating nursing staff on appropriately assessing pain in ICU patients. Integrating a palliative care team within the community hospital is also recommended. 
The implementation of the Care and Communication Bundle should be phased out at this current time at the local community hospital. As results of this study were inconclusive, to continue the implementation of the Care and Communication Bundle it would not be feasible. Once a palliative care team is re-instated at the local community hospital, implementation of the Care and Communication Bundle should be readdressed. There are no ongoing evaluations that need to be completed outside the scope of the DNP project. Future phases of the implementation of the Care and Communication Bundle could include providers addressing four of the nine quality measures upon admission. By doing so it aligns with the organizations strategic plan of providing the best, most effective and affordable care to the patients that are served at the local community hospital.

\section{Application Recommendations}

For the Care and Communication Bundle to be completely successful in implementation, the project location should have a palliative care team, and all electronic medical records. The implementation of the Care and Communication Bundle requires many hours, which would be most appropriately done by a palliative care team member, and not solely nursing staff. Staff members not on a palliative care team may find implementing this project difficult. At this local community hospital, there are still paper charts. Therefore, a fully electronic medical system, with pop-up reminders is highly recommended. With the appropriate team and electronic medical records, this project can be implemented at a larger scale than this DNP capstone project.

\section{Conclusions}

The overall aim of this project was to evaluate if the implementation of the Care and Communication Bundle increased the implementation of nine quality measures in the ICU at the 
local community hospital. This was partially achieved as evaluated by the nine objectives aligned with the nine quality palliative measures of the Care and Communication Bundle. Four of nine quality, palliative measures significantly improved after the implementation of the Care and Communication Bundle. This project illustrates that palliative care improvement can be achieved through project implementation, but continues to be a challenge in WV ICUs, and throughout the United States.

\section{Attainment of DNP Essentials}

\section{Essential I: Scientific Underpinnings for Practice}

This doctoral project focused on implementation of the Care and Communication Bundle which integrated nursing knowledge including ethics. This project developed and evaluated a new practice approach in the ICU using a theoretical framework. Successful change post project implementation supported DNP essential I by promoting nursing disciplines in regards to holistic patient centered care.

\section{Essential II: Organizational and Systems Leadership for Quality Improvement and Systems Thinking}

DNP Essential II aligns with this change project in the aspect that the local community hospital lost their palliative care team. This project's implementation improved the organizational and systems leadership to improve patient quality palliative care. This project developed and created a delivery approach (Care and Communication Bundle), to address the patient's needs at this local community hospital.

\section{Essential III: Clinical Scholarship and Analytical Methods for Evidence-Based Practice}


DNP Essential III aligns with this project from multiple aspects including: using analytical methods to search existing literature to evaluate best practice, designing a doctoral level change project, implementing the project, evaluating findings of project, and lastly apply relevant findings to develop practice guidelines at the community hospital. Each aspect of this DNP essential was met by completing this project.

\section{Essential IV: Information Systems/Technology and Patient Care Technology for the Improvement and Transformation of Health Care}

The process of creating designing, analyzing, implementing, and evaluating this implementation project aligns with the DNP essential IV. The APRN was prepared sufficiently in all aspects of implementation of project which including applying a budget and utilizing tools (Care and Communication Bundle) to improve and transform patient care.

\section{Essential V: Health Care Policy for Advocacy in Health Care}

The ultimate goal of the Care and Communication Bundle was to increase the timely implementation/documentation of the nine quality measures specific to this project. By doing so, this allowed for the patient's wishes to be upheld and prevented the patient from receiving care that may not have been desired. Therefore, implementation of this project at the local community hospital was important to promote staff members to advocate for patients in regards to palliative care.

\section{Essential VI: Interprofessional Collaboration for Improving Patient and Population Health}

\section{Outcomes}

The Care and Communication Bundle aligns with DNP essential VI in regards to the interprofessional collaboration that the bundle created. It required multiple interprofessional groups to collaborate to complete the bundle and to create change in health care at this local 
community hospital. Interprofessional collaboration in the implementation of this project was crucial for the patient to have the most effective, efficient, timely, and patient centered care.

\section{Essential VII: Clinical Prevention and Population Health for Improving the Nation's}

\section{Health}

DNP essential VII correlated with the implementation to this project in regards to understanding the impact that chronic diseases and clinical prevention has on the patients that arrive to the ICU. Clinical prevention starting at the earliest level is best practice, but unfortunately doesn't always occur. Therefore, patients with uncontrolled chronic diseases are ending up in the ICU without a plan of care. The implementation of this project on all ICU patients allowed for patients with chronic diseases earlier palliative care discussions, ultimately improving the Nation's Health.

\section{Essential VIII: Advanced Nursing Practice}

DNP essential VIII allowed nurses and APRNs to advocate for their critically ill patients, and promotes the utilization of critical thinking skills. These aspects of nursing demonstrated advanced nursing practice at the highest level. This project also promoted the APRN to guide, mentor, and support other nurses to implement the Care and Communication Bundle. 


\section{References:}

ALMouaalamy, N., Heckman, G., \& Lewis, D. (2018). Documentation of Code Status for Elderly Patients Admitted to a Hospital. Archives of Medicine, 10(2). doi: $10.21767 / 1989-5216.1000261$

American Association of Colleges of Nursing. (2006). The essentials of doctoral education for advanced nursing practice. Washington, DC. Retrieved from https://www.aacnnursing.org/DNP/DNP-Essentials

Average Income by State, Median, Top \& Percentiles. (2019). Retrieved 4 April 2020, from https://dqydj.com/average-income-by-state-median-top-percentiles/

Chick-fil-A - Home of the Original Chicken Sandwich. (2019). Retrieved from https://www.chick-fil a.com

Choosing a statistical test - FAQ 1790 - GraphPad. (2019). Retrieved from https://www.graphpad.com/support/faqid/1790/

Constantine, L. (2016). Evaluation of a Multifaceted Educational Intervention to Improve Palliative Care in the Intensive Care Unit. Journal Of Hospice \& Palliative Nursing, 18(6), 519-525. doi: 10.1097/njh.0000000000000288

Findings State Rankings | 2019 Senior Report. (2020). Retrieved 4 April 2020, from https://www.americashealthrankings.org/learn/reports/2019-senior-report/findings-state rankings

Gade, G., Venohr, I., Conner, D., McGrady, K., Beane, J., \& Richardson, R. et al. (2008). Impact of an Inpatient Palliative Care Team: A Randomized Controlled Trial. Journal Of Palliative Medicine, 11(2), 180-190. doi: 10.1089/jpm.2007.0055 
Libraries, W. (2019). Printing, Copying, and Scanning | WVU Libraries. Retrieved from https://lib.wvu.edu/services/printing/

Marx, A. (2019). Survey Analysis in 2019: How to Analyze Results [3 Examples]. Retrieved from https://getthematic.com/insights/analyze-survey-data-survey-analysis/

Mission, Vision \& Values | Mon Health Medical Center. (2019). Retrieved from https://www.mongeneral.com/main/mission-and-values

Nelson, J., Mulkerin, C., Adams, L. and Pronovost, P. (2006). Improving comfort and communication in the ICU: a practical new tool for palliative care performance measurement and feedback. Quality and Safety in Health Care, 15(4), pp.264-271.

Registered Nurse Salary in West Virginia. (2019). Retrieved from https://www.topregisterednurse.com/salary/west-virginia/

Salary.com, S. (2019). Hourly wage for Nurse Practitioner jobs in Morgantown, WV | Salary.com. Retrieved from https://www.salary.com/research/salary/benchmark/nurse practitioner-hourly-wages/morgantown-wv

Setting SMARTER Goals in 7 Easy Steps. (2019). Retrieved from https://www.projectsmart.co.uk/setting-smarter-goals-in-7-easy-steps.php

Centers for Disease Control National Center for Health Statistics. (2017). Stats of the State of West Virginia. Retrieved 4 April 2020, from https://www.cdc.gov/nchs/pressroom/states/westvirginia/westvirginia.htm SUPPORT Principal Investigators. (1996). A controlled trial to improve care for seriously ill hospitalized patients. JAMA, 275(16):1232.

U.S. Census Bureau QuickFacts: Monongalia County, West Virginia. (2020). Retrieved 4 April 2020, from https://www.census.gov/quickfacts/monongaliacountywestvirginia 
U.S. Census Bureau QuickFacts: West Virginia. (2020). Retrieved 4 April 2020, from https://www.census.gov/quickfacts/WV

Vuong, C., Kittelson, S., McCullough, L., Yingwei, Y., \& Hartjes, T. (2019). Implementing primary palliative care best practices in critical care with the Care and Communication Bundle. BMJ Open Quality, 8(3), e000513. doi: 10.1136/bmjoq-2018-000513

Wessman, B., Sona, C., \& Schallom, M. (2016). Improving Caregivers' Perceptions Regarding Patient Goals of Care/End-of-Life Issues for the Multidisciplinary Critical Care Team. Journal of Intensive Care Medicine, 32(1), 68-76. doi: 10.1177/0885066615606063

West Virginia Salaries - Average Salary in West Virginia - PayScale. (2019). Retrieved 22 July 2019, from https://www.payscale.com/research/US/State=West_Virginia/Salary

West Virginia Vital Statistics, (2015) Retrieved 4 April 2020, from http://www.wvdhhr.org/bph/hsc/pubs/vital/2015/Vital2015_Minus_Divorce_Data.pdf Wojciechowski, E., Pearsall, T., Murphy, P., \& French, E. (2016). A Case Review: Integrating Lewin's Theory with Lean's System Approach for Change. Online journal of issues in nursing, 21(2), 4. https://doi.org/10.3912/OJIN.Vol21No02Man04 
Table 1

Project Budget Personnel Costs

\begin{tabular}{|c|c|c|c|}
\hline Total & & & \\
\hline$\$ 26,118.00$ & & & \\
\hline Role & Department & Hours Spent & Base Salary \\
\hline Co-PI (myself) & Student & $7 \mathrm{hrs} \times 12$ weeks & $\$ 47 \mathrm{hr}=\$ 3,948$ \\
\hline Capstone Chair (PI) & School of Nursing & $1 \mathrm{hr} \times 12$ weeks & $\$ 50 \mathrm{hr}=\$ 600$ \\
\hline Project Manager MD & Project Site & $1 \mathrm{hr} \times 12$ weeks & $\begin{array}{l}\$ 150 \mathrm{hr}= \\
\$ 1,800\end{array}$ \\
\hline ICU Director & Project Site & 6 hours & $\$ 75 \mathrm{hr}=\$ 450$ \\
\hline ICU Staff Nurses (5) & Project Site & $7 \mathrm{hrs} \times 12$ weeks & $\begin{array}{l}\$ 30 \mathrm{hr}= \\
\$ 12,600\end{array}$ \\
\hline $\begin{array}{l}\text { Care Management } \\
\text { Staff (2) }\end{array}$ & Project Site & $7 \mathrm{hrs} \times 12$ weeks & $\$ 30 \mathrm{hr}=\$ 5,040$ \\
\hline Pastoral Care Staff & Project Site & 7hrs x 12 weeks & $\$ 20 \mathrm{hr}=\$ 1,680$ \\
\hline $\begin{array}{l}\text { *Total human costs } \\
\text { were integrated into } \\
\text { current roles of the } \\
\text { persons listed above }\end{array}$ & & & \\
\hline
\end{tabular}


Table 2

Project Budget Equipment/Supplies Costs

\begin{tabular}{|l|l|l|l|}
\hline Total & & & \\
\hline $\mathbf{\$ 2 9 5 . 7 4}$ & & & \\
\hline Item & Quantity & Cost Each & Total Cost with 0.06\% \\
\hline SPSS Software & 1 & & Sales Tax \\
\hline Paper & & $\$ 90.00$ & $\$ 95.40$ \\
\hline Ink & 1 & $\$ 6.00$ & $\$ 6.36$ \\
\hline Professional Poster & 3 & $\$ 30$ & $\$ 63.60$ \\
\hline Catered Lunch & 1 & $\$ 12$ & $\$ 38.16$ \\
\hline
\end{tabular}


Table 3

Quality Measure Frequencies both Pre-Intervention and Post-Intervention as Documented by Day 1

\begin{tabular}{|c|c|c|c|c|}
\hline Quality & Pre-Intervention & Post- Intervention & Chi Square & Sig. \\
\hline Measure & Frequency/Percent & Frequency/Percent & Value & \\
\hline \multicolumn{5}{|c|}{ Advanced Directives } \\
\hline YES & $Y=15-50 \%$ & $Y=18-60 \%$ & 0.61 & $\begin{array}{l}p= \\
0.44\end{array}$ \\
\hline NO & $\mathrm{N}=15-50 \%$ & $\mathrm{~N}=12-40 \%$ & & \\
\hline \multicolumn{5}{|c|}{ MPOA or Surrogate } \\
\hline YES & $Y=10-33 \%$ & $Y=17-56 \%$ & 3.3 & $\begin{array}{l}p= \\
0.07\end{array}$ \\
\hline NO & $\mathrm{N}=20-67 \%$ & $\mathrm{~N}=13-43 \%$ & & \\
\hline \multicolumn{5}{|c|}{ Resuscitation Status } \\
\hline $\begin{array}{l}\text { YES, FULL } \\
\text { CODE }\end{array}$ & $\mathrm{Y} / \mathrm{A}=13-44 \%$ & $\mathrm{Y} / \mathrm{A}=17-56 \%$ & 1.36 & $\begin{array}{l}p= \\
0.24\end{array}$ \\
\hline YES, DNR & $\mathrm{Y} / \mathrm{B}=7-23 \%$ & $\mathrm{Y} / \mathrm{B}=7-23 \%$ & & \\
\hline NO & $\mathrm{N}=10-33 \%$ & $\mathrm{~N}=6-20 \%$ & & \\
\hline \multicolumn{5}{|l|}{$\begin{array}{l}\text { Patient } \\
\text { Leaflet }\end{array}$} \\
\hline YES & $\mathrm{Y}=0 \%$ & $Y=23-76 \%$ & & \\
\hline $\mathrm{NO}$ & $\mathrm{N}=0 \%$ & $\mathrm{~N}=7-23 \%$ & & \\
\hline
\end{tabular}


Table 4

Quality Measure Frequencies both Pre-Intervention and Post-Intervention as Documented by

Day 3

\begin{tabular}{|c|c|c|c|c|}
\hline Quality Measure & $\begin{array}{l}\text { Pre-Intervention } \\
\text { Frequency/Percent }\end{array}$ & $\begin{array}{l}\text { Post- Intervention } \\
\text { Frequency/Percent }\end{array}$ & $\begin{array}{l}\text { Chi Square } \\
\text { Value }\end{array}$ & Sig. \\
\hline Care Managemen & & & & \\
\hline $\begin{array}{l}\text { Yes with patient } \\
\text { only }\end{array}$ & $\mathrm{Y} / \mathrm{A}=8-26 \%$ & $\mathrm{Y} / \mathrm{A}=15-50 \%$ & 5.71 & $\begin{array}{l}p= \\
0.02\end{array}$ \\
\hline $\begin{array}{l}\text { Yes with family } \\
\text { only }\end{array}$ & $\mathrm{Y} / \mathrm{B}=2-7 \%$ & $\mathrm{Y} / \mathrm{B}=4-13 \%$ & & \\
\hline $\begin{array}{l}\text { Yes with pt and } \\
\text { family }\end{array}$ & $\mathrm{Y} / \mathrm{C}=4-13 \%$ & $\mathrm{Y} / \mathrm{C}=4-13 \%$ & & \\
\hline No & $\mathrm{N}=16-53 \%$ & $\mathrm{~N}=7-23 \%$ & & \\
\hline $\begin{array}{l}\text { Spiritual Support } \\
\text { Offered }\end{array}$ & & & & \\
\hline $\begin{array}{l}\text { Yes with patient } \\
\text { only }\end{array}$ & $\mathrm{Y} / \mathrm{A}=4-13 \%$ & $\mathrm{Y} / \mathrm{A}=15-50 \%$ & 13.3 & $\begin{array}{l}\mathrm{p}< \\
0.01\end{array}$ \\
\hline $\begin{array}{l}\text { Yes with family } \\
\text { only }\end{array}$ & $\mathrm{Y} / \mathrm{B}=2-7 \%$ & $Y / B=5-16 \%$ & & \\
\hline $\begin{array}{l}\text { Yes with pt and } \\
\text { family }\end{array}$ & $\mathrm{Y} / \mathrm{C}=4-13 \%$ & $\mathrm{Y} / \mathrm{C}=4-13 \%$ & & \\
\hline
\end{tabular}




\begin{tabular}{|l|l|l|l|l|}
\hline No & $\mathrm{N}=20-67 \%$ & $\mathrm{~N}=6-20 \%$ & & \\
\hline
\end{tabular}


Table 5

Quality Measure Frequencies both Pre-Intervention and Post-Intervention as Documented by Day 5

\begin{tabular}{|c|c|c|c|c|}
\hline Quality Measure & $\begin{array}{l}\text { Pre-Intervention } \\
\text { Frequency/Percent }\end{array}$ & $\begin{array}{l}\text { Post- Intervention } \\
\text { Frequency/Percent }\end{array}$ & $\begin{array}{l}\text { Chi } \\
\text { Square } \\
\text { Value }\end{array}$ & Sig. \\
\hline $\begin{array}{l}\text { Interdisciplinary } \\
\text { Family Meeting }\end{array}$ & & & & \\
\hline $\begin{array}{l}\text { Yes with patient } \\
\text { only }\end{array}$ & $\mathrm{Y} / \mathrm{A}=2-7 \%$ & $\mathrm{Y} / \mathrm{A}=8-26 \%$ & 8.3 & $\begin{array}{l}\mathrm{p}< \\
0.01\end{array}$ \\
\hline $\begin{array}{l}\text { Yes with family } \\
\text { only }\end{array}$ & $\mathrm{Y} / \mathrm{B}=2-7 \%$ & $\mathrm{Y} / \mathrm{B}=4-13 \%$ & & \\
\hline $\begin{array}{l}\text { Yes with pt and } \\
\text { family }\end{array}$ & $\mathrm{Y} / \mathrm{C}=3-10 \%$ & $Y / C=6-20 \%$ & & \\
\hline No & $\mathrm{N}=23-76 \%$ & $\mathrm{~N}=12-40 \%$ & & \\
\hline
\end{tabular}


PALLIATIVE CARE IN THE ICU

Table 6

Nurse Survey Results

\begin{tabular}{|c|c|c|c|c|c|}
\hline NURSE: & Q\#1 & $\mathrm{Q} \# 2$ & Q\#3 & Q\#4 & $\mathrm{Q \# 5}$ \\
\hline NURSE 001 & $\mathrm{Y}$ & $\mathrm{Y}$ & $\mathrm{Y}$ & $\mathrm{Y}$ & $\mathrm{Y}$ \\
\hline NURSE 002 & $\mathrm{Y}$ & $\mathrm{Y}$ & $Y$ & $\mathrm{Y}$ & Y \\
\hline NURSE 003 & $\mathrm{Y}$ & $\mathrm{Y}$ & $Y$ & $\mathrm{~N}$ & $\mathrm{Y}$ \\
\hline NURSE 004 & $\mathrm{Y}$ & $\mathrm{Y}$ & $Y$ & $\mathrm{Y}$ & $\mathrm{Y}$ \\
\hline NURSE 005 & $\mathrm{~N}$ & $\mathrm{Y}$ & $\mathrm{N}$ & $\mathrm{N}$ & $\mathrm{N}$ \\
\hline NURSE 006 & $\mathrm{Y}$ & $\mathrm{Y}$ & $\mathrm{Y}$ & $\mathrm{Y}$ & $\mathrm{Y}$ \\
\hline NURSE 007 & $\mathrm{Y}$ & $\mathrm{Y}$ & $Y$ & $\mathrm{Y}$ & Y \\
\hline NURSE 008 & $\mathrm{Y}$ & $\mathrm{Y}$ & $Y$ & $\mathrm{Y}$ & $\mathrm{Y}$ \\
\hline NURSE 009 & $\mathrm{Y}$ & $\mathrm{Y}$ & $\mathrm{Y}$ & $\mathrm{N}$ & $\mathrm{Y}$ \\
\hline NURSE 010 & $\mathrm{Y}$ & $\mathrm{Y}$ & $\mathrm{Y}$ & $\mathrm{Y}$ & $\mathrm{Y}$ \\
\hline NURSE 011 & $\mathrm{Y}$ & $\mathrm{Y}$ & $\mathrm{Y}$ & $\mathrm{Y}$ & $\mathrm{Y}$ \\
\hline NURSE 012 & $\mathrm{Y}$ & $\mathrm{Y}$ & $\mathrm{Y}$ & $\mathrm{Y}$ & $\mathrm{Y}$ \\
\hline NURSE 013 & $\mathrm{~N}$ & $\mathrm{Y}$ & $\mathrm{N}$ & $\mathrm{N}$ & $\mathrm{Y}$ \\
\hline NURSE 014 & $\mathrm{Y}$ & $\mathrm{Y}$ & $\mathrm{Y}$ & $\mathrm{Y}$ & $\mathrm{Y}$ \\
\hline NURSE 015 & $\mathrm{Y}$ & $\mathrm{Y}$ & $\mathrm{Y}$ & $\mathrm{Y}$ & $\mathrm{Y}$ \\
\hline NURSE 016 & $\mathrm{Y}$ & $\mathrm{Y}$ & $\mathrm{Y}$ & $\mathrm{N}$ & $\mathrm{Y}$ \\
\hline NURSE 017 & $\mathrm{~N}$ & $\mathrm{Y}$ & $\mathrm{Y}$ & $\mathrm{Y}$ & $\mathrm{Y}$ \\
\hline NURSE 018 & $\mathrm{Y}$ & $\mathrm{Y}$ & $\mathrm{Y}$ & $\mathrm{Y}$ & $\mathrm{Y}$ \\
\hline NURSE 019 & $\mathrm{~N}$ & $\mathrm{Y}$ & $\mathrm{N}$ & $\mathrm{N}$ & $\mathrm{N}$ \\
\hline NURSE 020 & $\mathrm{Y}$ & $\mathrm{Y}$ & $\mathrm{Y}$ & $\mathrm{Y}$ & $\mathrm{Y}$ \\
\hline
\end{tabular}




\begin{tabular}{|l|l|l|l|l|l|}
\hline NURSE 021 & $\mathrm{Y}$ & $\mathrm{Y}$ & $\mathrm{Y}$ & $\mathrm{Y}$ & $\mathrm{Y}$ \\
\hline NURSE 022 & $\mathrm{Y}$ & $\mathrm{Y}$ & $\mathrm{Y}$ & $\mathrm{Y}$ & $\mathrm{Y}$ \\
\hline NURSE 023 & $\mathrm{Y}$ & $\mathrm{Y}$ & $\mathrm{Y}$ & $\mathrm{Y}$ & $\mathrm{Y}$ \\
\hline NURSE 024 & $\mathrm{Y}$ & $\mathrm{Y}$ & $\mathrm{Y}$ & $\mathrm{Y}$ & $\mathrm{Y}$ \\
\hline NURSE 025 & $\mathrm{Y}$ & $\mathrm{Y}$ & $\mathrm{Y}$ & $\mathrm{Y}$ & $\mathrm{Y}$ \\
\hline TOTALS & $\mathrm{Y}=21-84 \%$ & $\mathrm{Y}=25$ & $\mathrm{Y}=22-88 \%$ & $\mathrm{Y}=19-76 \%$ & $\mathrm{Y}=25$ \\
\hline & $\mathrm{N}=4-16 \%$ & $\mathrm{~N}=0$ & $\mathrm{~N}=3-12 \%$ & $\mathrm{~N}=6-24 \%$ & $\mathrm{~N}=0$ \\
\hline
\end{tabular}


The West Virginia Center for Endof-Life Care Offers the Following

Resources and Services:

Advance Directives

- Frequently Asked Questions Booklet about the Living Will and Medical Power of Attorney forms including the forms

- Do Not Resuscitate card brochure

- POST form brochure

Information about End-of-Life Care

- Palliative care brochure

- Hospice Services in the Nursing Home brochure

- "Hard Choices for Loving People" booklet

- Organ donation brochure

- Information about feeding tubes

Forms mailed to physicians or other health care providers for patient care

- Do Not Resuscitate card

- POST form

- Health Care Surrogate Checklist

- Pain Management card

WV Center for End-of-Life Care 1195 Health Sciences North PO Box 9022

Morgantown, WV $\mathbf{6} 6506-9022$

www.wvendoflie, $\alpha \mathrm{g}$

1-877-209-8086

\section{Palliative} Care

Comfort and Support for Persons with Serious Illness and Their Familiesf
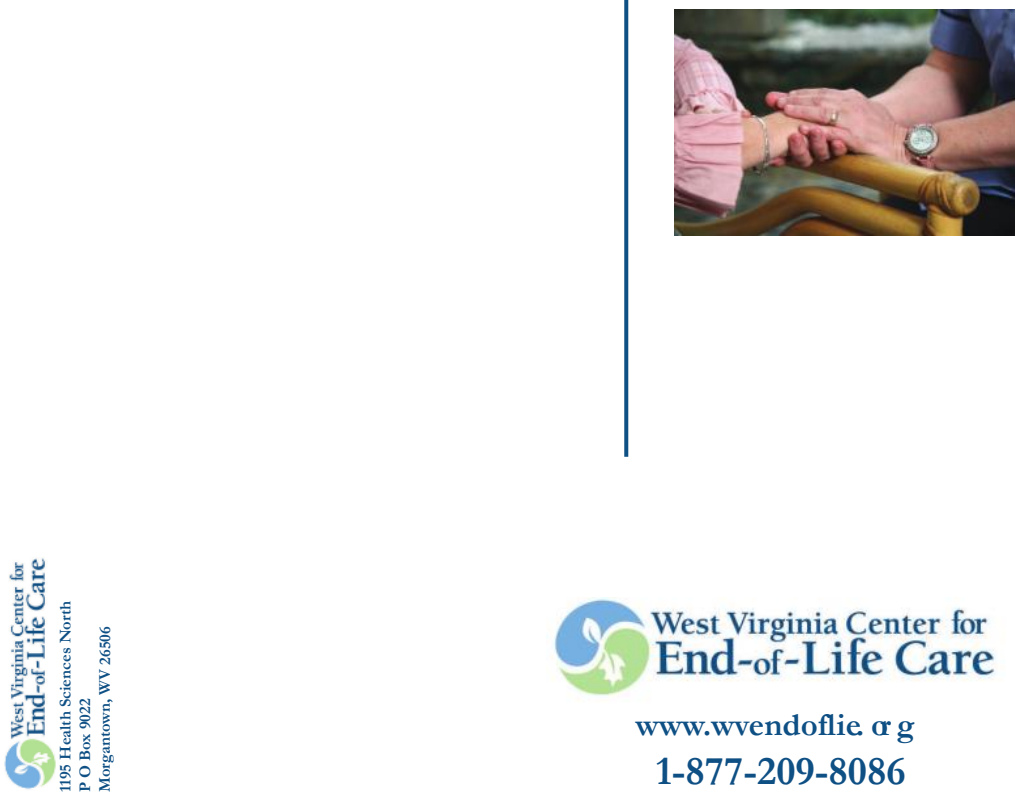

West Virginia Center for End-of-Life Care www.wvendoflie. $\alpha \mathrm{g}$ 1-877-209-8086

Figure 1. Palliative Care Pamphlet. Adapted from West Virginia Center for End-of-Life Care. http://wvendoflife.org/media/1080/pc-brochure-2009.pdf 
"Palliative" (pa-lè-ā-tiv) means to relieve or reduce pain and discomfort. The goal of palliative care is to prevent and relieve suffering, and to support the best possible quality of life for patients and their families, regardless of their stage of disease or the need for other therapies,

in accordance with their values and preferences.

Palliative Care

- provides relief from pain and other symptoms

- comforts whether one has vears, months or days to live

- is appropriate at any age

- helps with the emotional and spiritual needs of patients and families

- affirms life and regards dying as a normal process

- neither hastens nor postpones death

- offers a support system to help patients live as actively as possible as long as possible

- offers a support system to help the family cope during the patient's illness and in their own grieving

"One of the hardest things in life is to watch a loved one suffer and feel an overwhelming sense of helplessness. Through palliative care the suffering and helplessness are replaced with a calming sense of peacefulness for both the patient and family."

Family member of patient receiving palliative care
Palliative Care Service

A palliative care team provides services from many different health care professionals for patients with life-limiting illnesses.

Palliative care services may be provided by:

- Hospitals

- Hospices

- Nursing homes

- Home health agencies

The team has special training in managing pain and other symptoms, and can help patients and families face difficl t deci sions .

For those not in the hospital, a palliative care service may be available to outpatients. The team can coordinate services with many other care providers such as home health agencies, hospices, and nursing homes. This service coordination provides extra support to patients and their families after they leave the hospital.

A Palliative Care Team May Include:

- Nurse Coordinator

- Physician

- Clinical social worker

- Pharmacist

- Psychiatrist

- Chaplain

- Physical therapist

- Occupational therapist

- Nutritionist

- Music therapist

- Speech therapist
Palliative Care Services Treat the Whole Person and May Include:

- Exploring patient goals and wishes

- Clarifying medication and treatments

- Promoting communication with the health care team

- Supporting the completion of an advance directive

- Discussing pain and symptom management and the meaning of comfort care

- Providing information and support regarding home care, skilled nursing, and hospice care

"Before palliative care consultation, $40 \%$ of patients reported severe pain. Within 48 hours after the palliative care consultation fewer than $8 \%$ still reported severe pain."

2008 West Virginia Palliative Care Network, Annual Reort

To fin out $\mathrm{m}$ re about pal liat i ve care services available throughout West Virginia call the

West Virginia Center for End-of-Life Care toll free at

1-877-209-8086

Figure 1. Palliative Care Pamphlet Cont. Adapted from West Virginia Center for End-of-Life Care. http://wvendoflife.org/media/1080/pc-brochure-2009.pdf 


\begin{tabular}{|c|c|}
\hline $\begin{array}{l}\text { West VirginiaU } \\
\text { school of NURSING }\end{array}$ & $\begin{array}{l}\text { Care and Communication Bundle Integration } \\
\text { Into the ICU } \\
\text { Angela S. Bohon, MSN, FNP-C }\end{array}$ \\
\hline 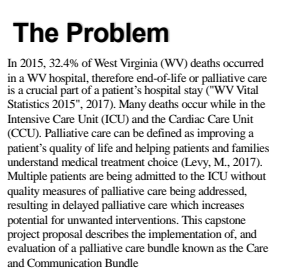 & 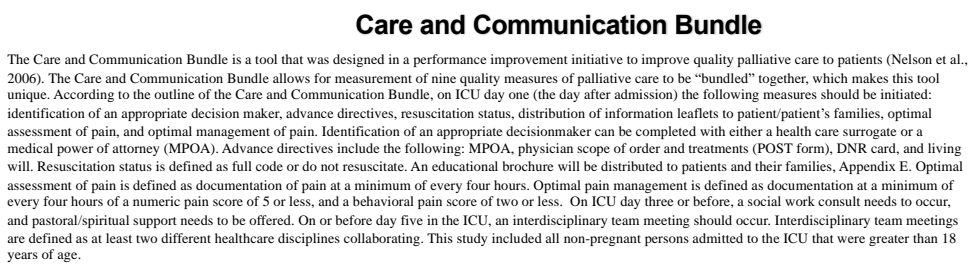 \\
\hline
\end{tabular}

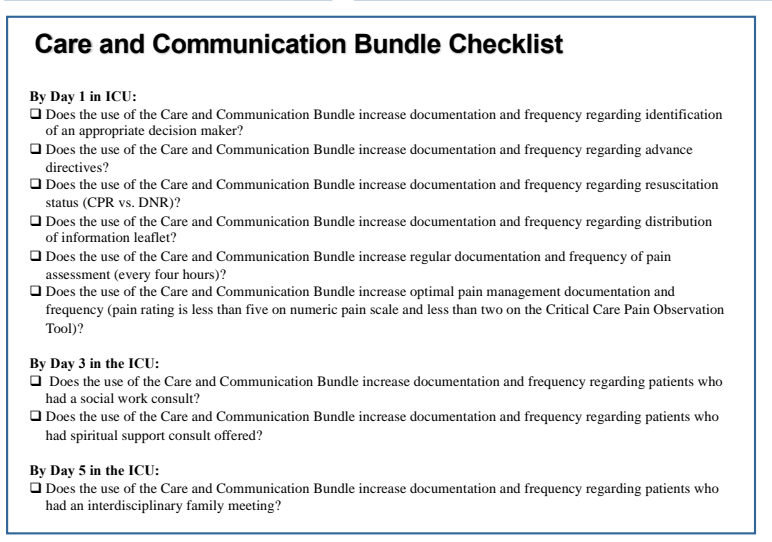

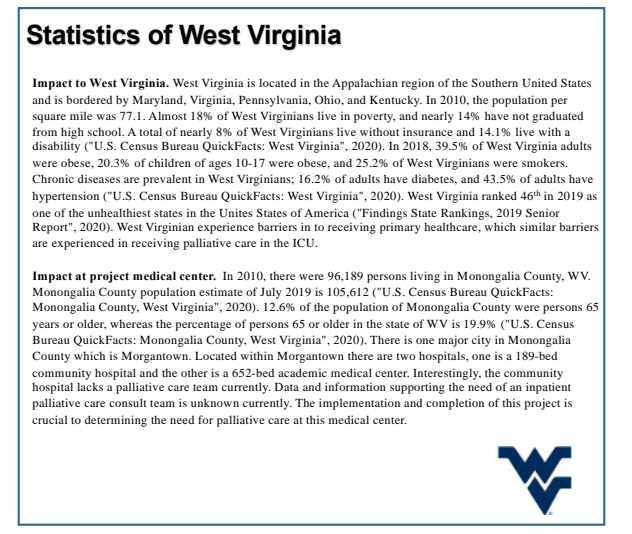

Figure 2. Poster Displayed in ICU. Care and Communication Bundle Integration into the ICU, by A. S. Bohon, 2019. 


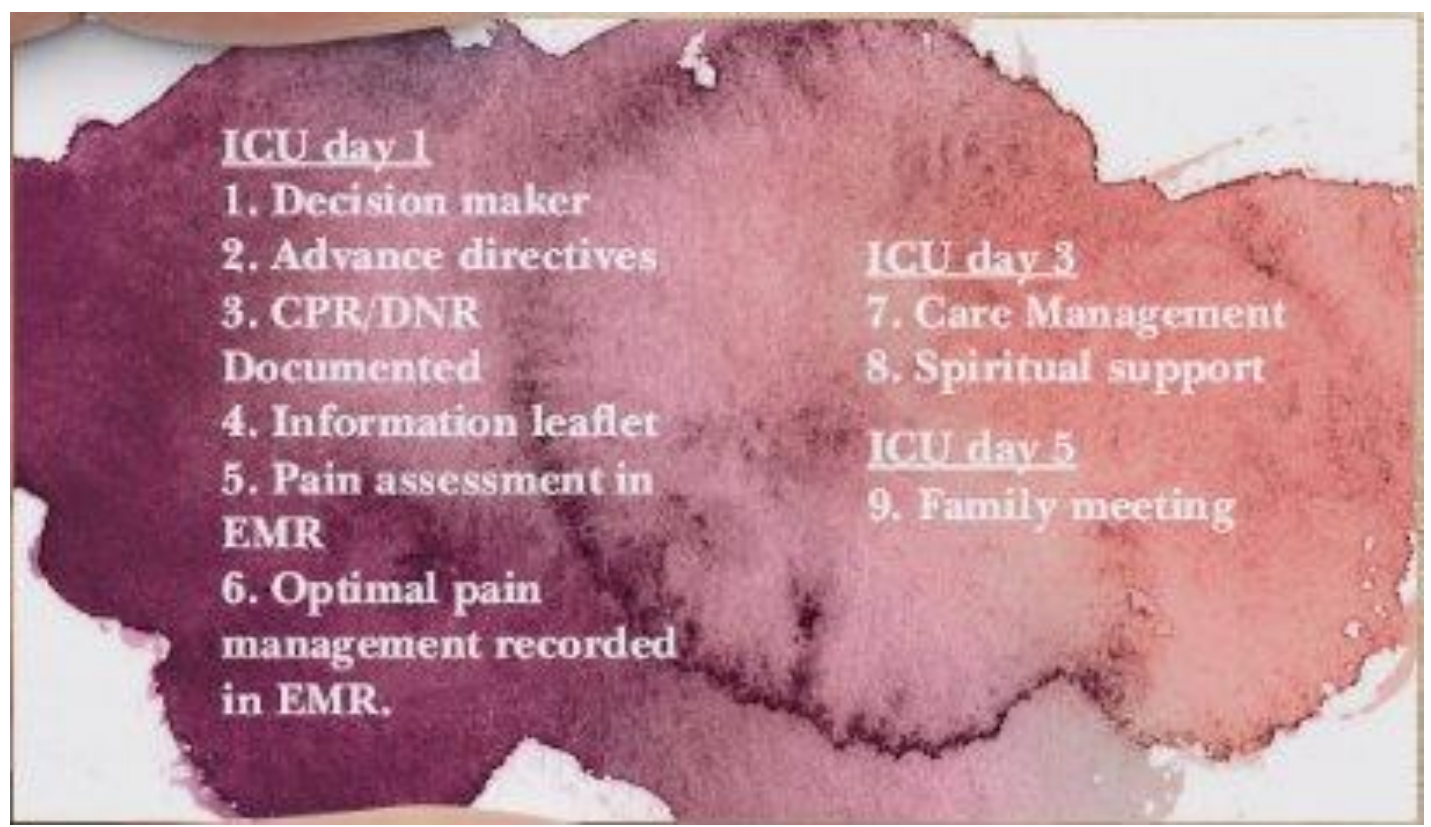

Figure 3. Pocket cards for nurses, by A. S. Bohon, 2019. 

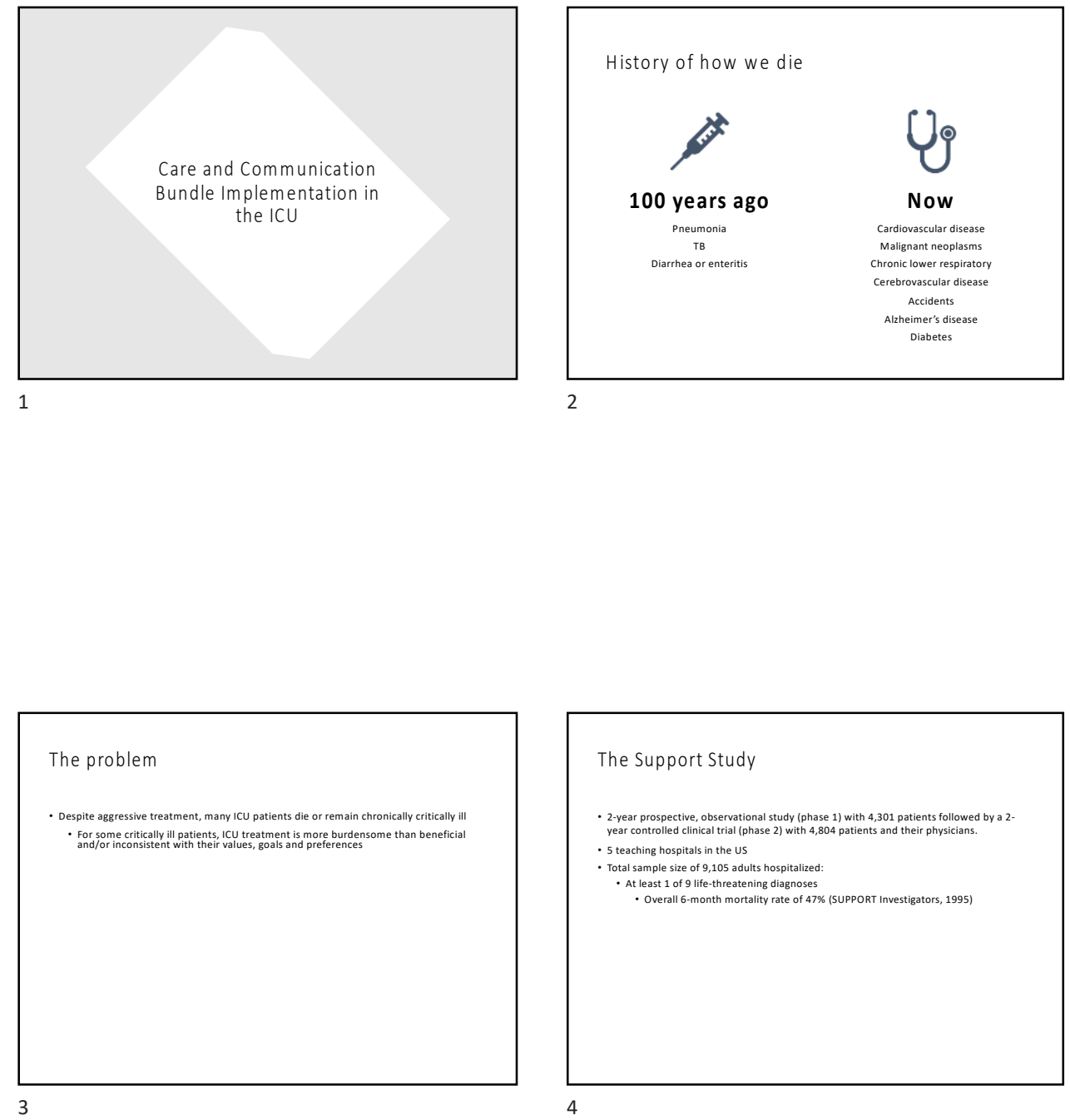

Figure 4. Educational handout to staff, by A. S. Bohon, 2019. 

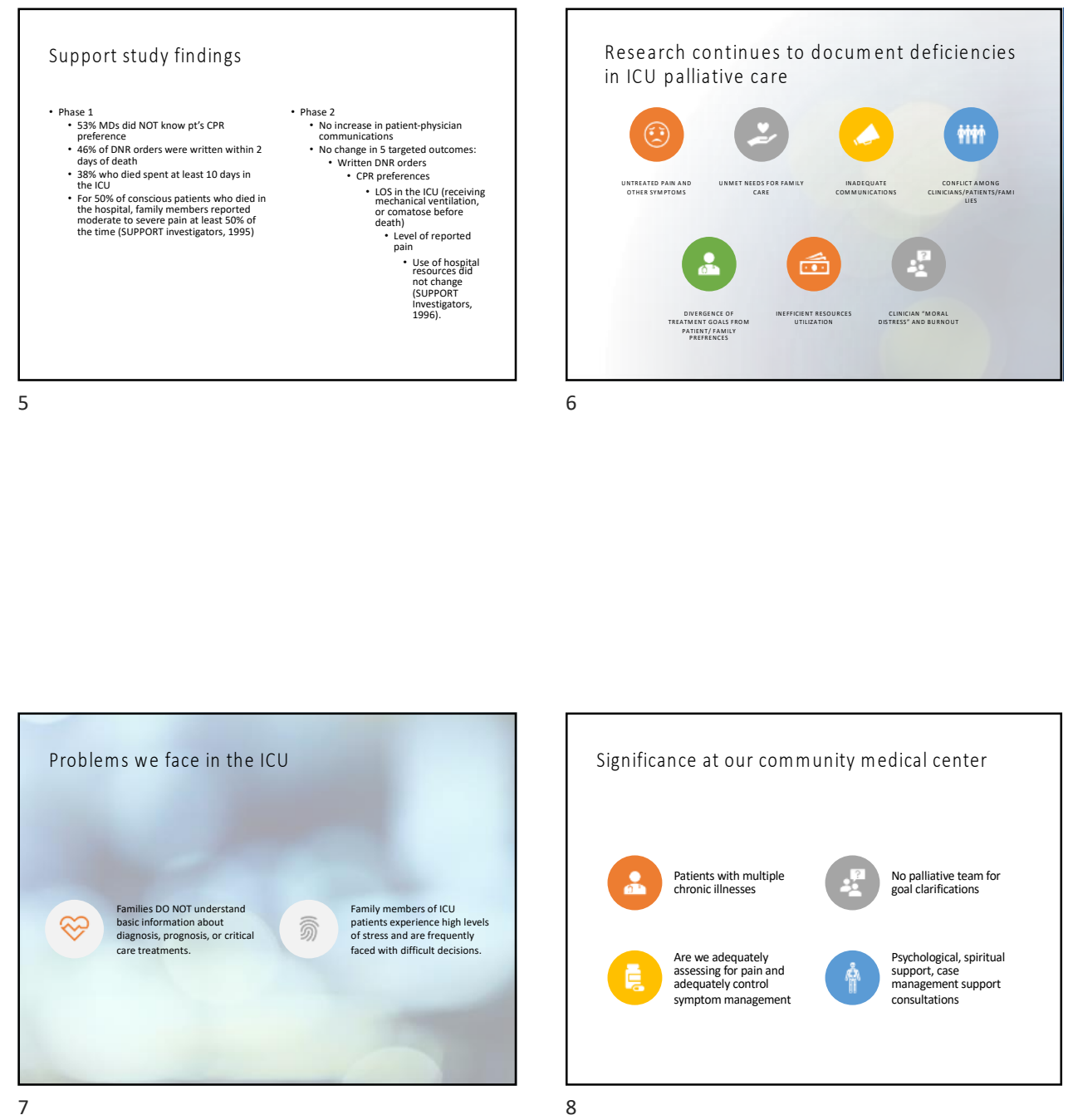

Figure 4. Educational handout to staff cont., by A. S. Bohon, 2019. 

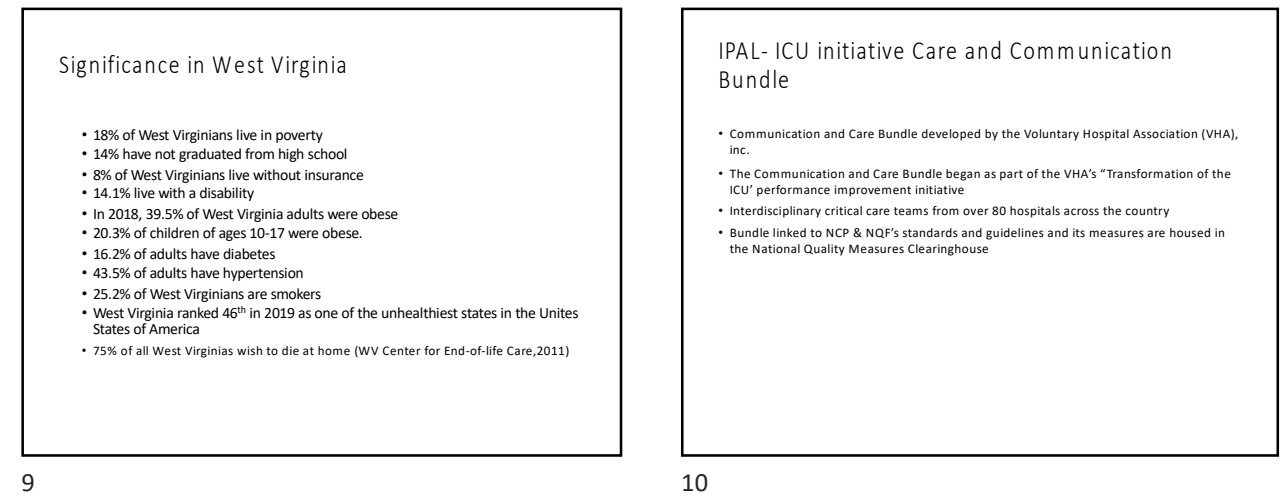

10

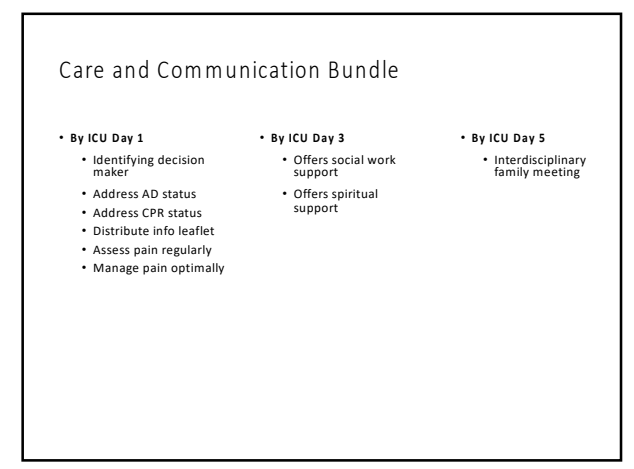

11

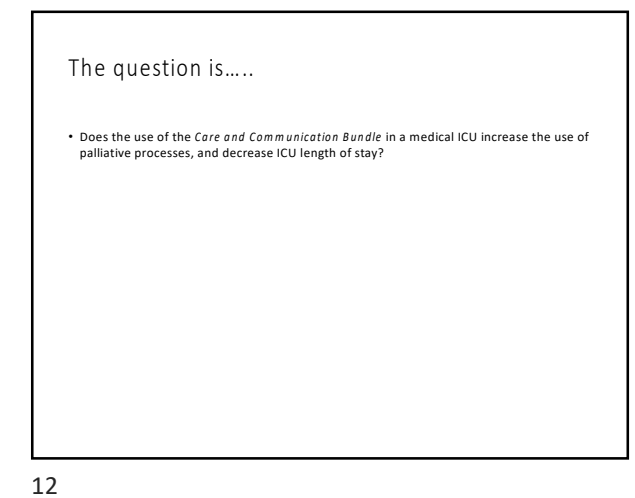

Figure 4. Educational handout to staff cont., by A. S. Bohon, 2019. 


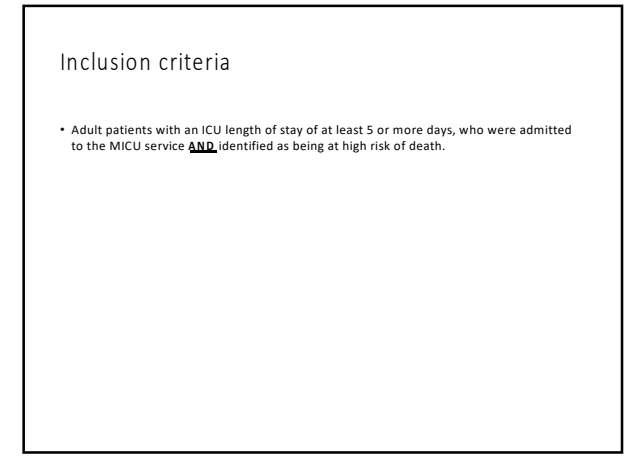

13

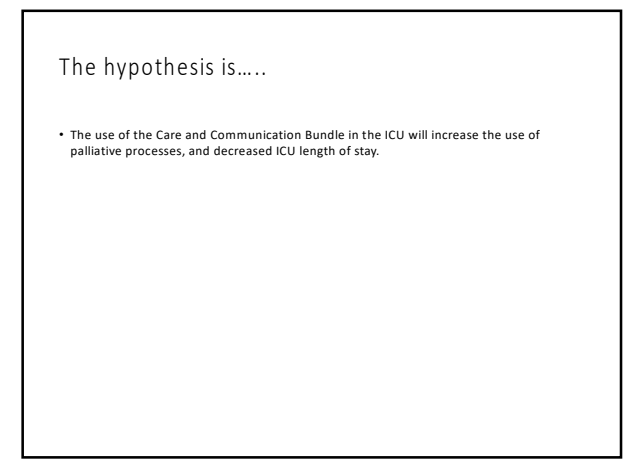

15

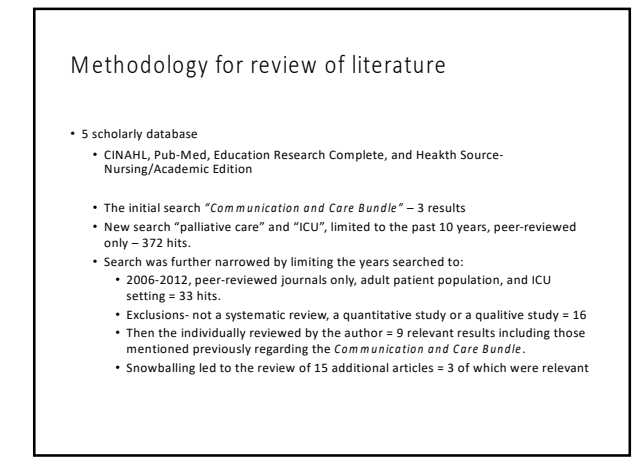

14

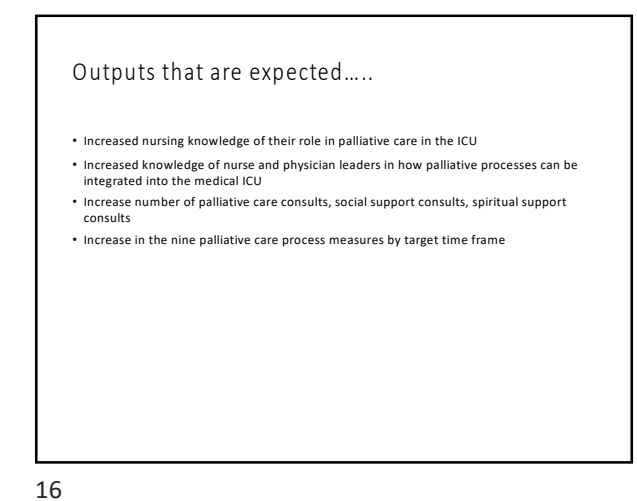

Figure 4. Educational handout to staff cont., by A. S. Bohon, 2019. 

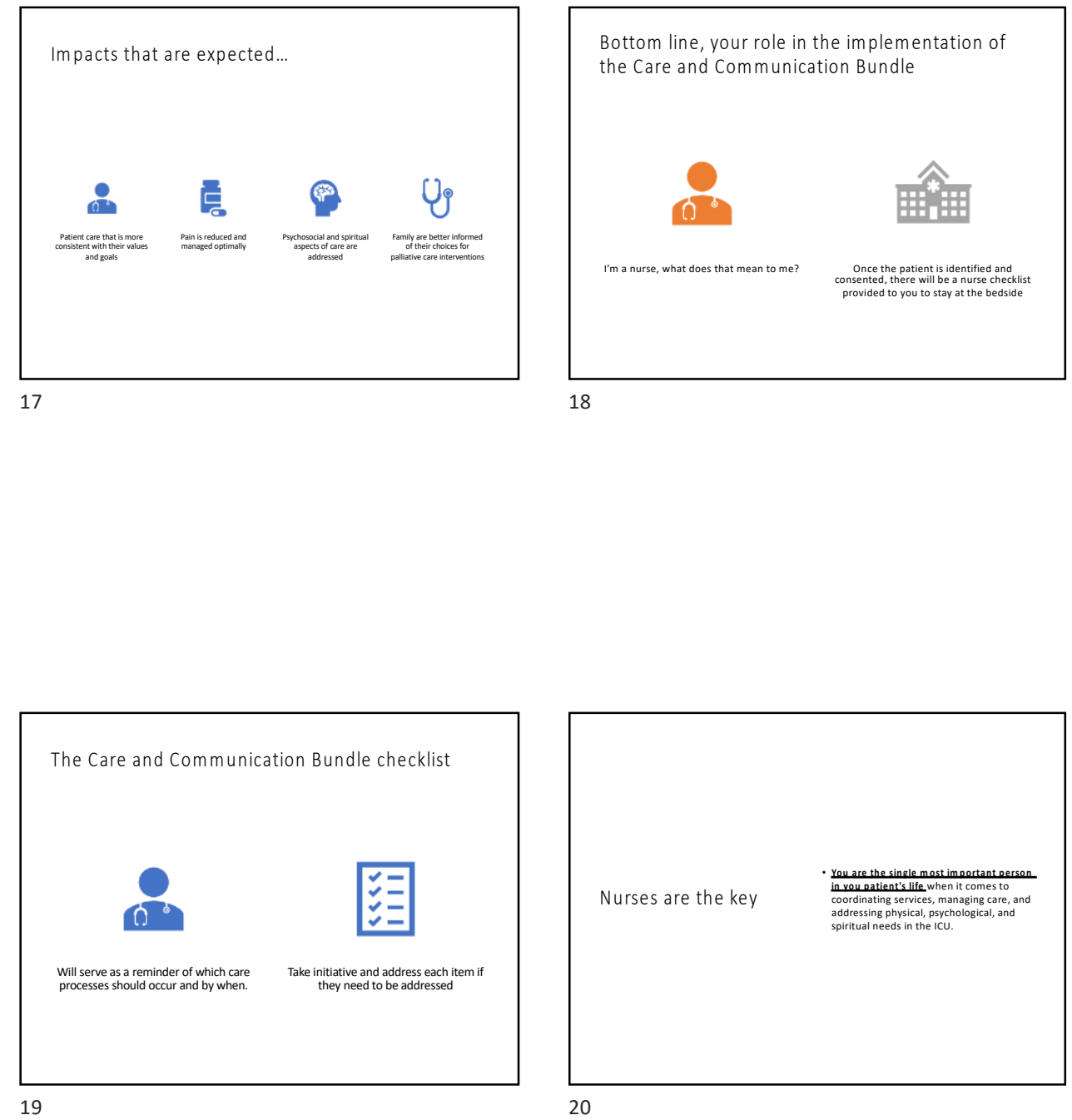

Figure 4. Educational handout to staff cont., by A. S. Bohon, 2019. 


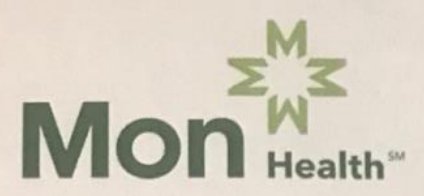

1200 JD Anderson Drive

Morgantown, WV 26505

304-598-1200

monhealth.com

October 7, 2019

\section{Dear Angela:}

I am pleased to offer my support and approval for you to conduct your DNP research at Mon Health Medical Center in the ICU and CCU. This approval is granted for the purposes of your study, "Evaluation of the Care and Communication Bundle to Improve Palliative Care in Intensive Care Units."

As you know, the next step is to secure approval from the Mon Health Medical Center IRB.

I'm looking forward to learning the results of your study and how Mon Health can use your findings to improve nursing practice.

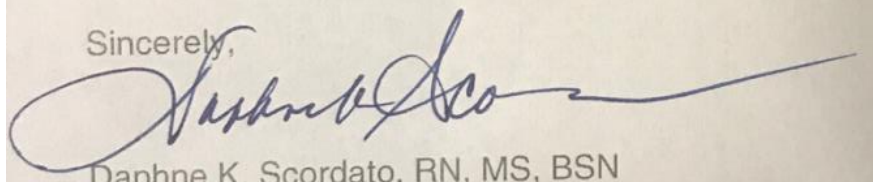

Chief Nursing Officer/ Vice- President, Patient Care Services

Figure 5. Site Support, by D. K. Scordato, 2019. 


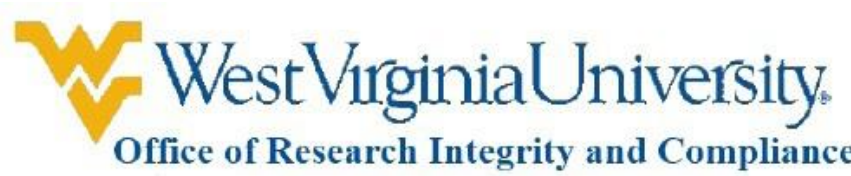

886 Chestnut Ridge Road Morgantown, WV 26506

Acknowledgement of Not Human Subjects Research

$12 / 03 / 2019$

To: Lori Constantine

From: WVU Office of Research Integrity \& Compliance

Protocol Type: NHSR / Flex

Approval Date: 12/03/2019

Submission Type: Initial

Expiration Date: 12/02/2024

Funding: N/A

WVU Protocol \#: 1909705184

Protocol Title: Evaluation of the Care and Communication Bundle to Improve Palliative Care in Intensive Care Units

The West Virginia University Institutional Review Board has determined that your protocol submission of protocol 1909705184 does not meet the definition of human subjects research, as provided in $45 \mathrm{CFR}$ 46. This determination was based on the following:

- Research means a systematic investigation designed to develop or contribute to generalizable knowledge. Most case reports and most oral histories are not generalizable and, therefore, not research. Many classroom projects, if not intended to be published, are also not considered research. Many quality improvement or program evaluation studies are not research.

The following documents were reviewed and acknowledged for use as part of this submission. Only the documents listed below may be used in the research. Please access and print the files in the Notes \& Attachments section of your approved protocol.

- IAA.pdf

- retrospective_protocol_template_MON final.ASB.docx

- IAA ? Individual Protocol ? WVU IRB of Record.docx

- HIPAA Waiver Form. ASB.docx

$\begin{array}{rr}\text { Protocol \#: } 1909705184 & \text { Phone: } 304-293-7073 \\ \text { FWA: 00005078 } & \text { Fax: 304-293-3098 } \\ \text { IORG: 0000194 } & \text { Email: IRB@ mail.wvu.edu }\end{array}$

Figure 6. IRB Letter, by WVU Office of Research Integrity \& Compliance, 2019. 


\section{Appendix A}

Nurses checklist for chart

There are nine palliative measures in the Care and Communication Bundle. These nine care processes, which are quality measures, should be documented in the medical record at key time frames.

ICU day 1 (the day after ICU admission)

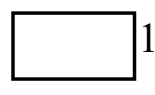

1. Identification of appropriate decision maker

2. Presence of advance directives

3. CPR status that is documented in the electronic medical record (EMR).

4. Documented distribution of information leaflet to patients and/or families in EMR.

5. Ongoing regular pain assessment in EMR. (Regular ongoing pain assessment is at minimum every 4 hours.)

6. Ongoing optimal pain management recorded in EMR. (Optimal pain management is defined by the patient scoring $<5$ on the numeric pain scale)

\section{ICU day 3}

7. Care Management contact documented in the EMR.

8. Spiritual support offered and documented in the EMR

\section{ICU day 5}

9. Interdisciplinary family meeting documented in the EMR 


\section{Appendix B}

Pre and Post Intervention Inclusion and Exclusion Criteria

\section{Inclusion Criteria}

Adult patients were admitted to the ICU service. The checked box or boxes is the defining reasons this patient met the inclusion criteria.

$\square \quad$ ICU admission

$\square \quad$ Age greater than 18yrs

\section{Exclusion Criteria}

$\square$ Pregnant persons

$\square \quad$ Persons less than 18 years old 


\section{Appendix C}

\section{Code Key for Chart Abstraction Tool}

Quality Measure \#1: MPOA/Surrogate documented: "Y"/"N"

Quality Measure \#2: Advance directives (AD) documented: "Y"/"N"

Quality Measure \#3: Resuscitation Status: "Y"/"N" If "Y" A) Full Code, B) DNR

Quality Measure \#4: Distribution/documentation of ICU info leaflet given: "Y"/"N"

Quality Measure \#5: Regular Pain Assessment: The number of 4-hour intervals eligible for pain assessment over the number of 4-hour intervals with pain assessment documented

Quality Measure \#6: Optimal Pain Management: The number of 4hour intervals with documented numerical pain value over the number of 4hour intervals with numeric pain scores <

5.

Quality Measure \#7: Documentation that Care Management was contacted either the patient or the family: "Y"/"N" If "Y" offered to A) patient, B) family, C) both

Quality Measure \#8: Documentation that Spiritual Support was offered: "Y"/"N" If "Y" offered to A) patient, B) family C) both

Quality Measure \#9: Interdisciplinary meeting held on/before Day 5: "Y"/"N"/"NA"/"Meeting Refused"; If "Y" meeting with A) patient, B) family C) both

LOS/Mortality: The number of ICU days at either discharge from the ICU or death. Mortality is "Y" for having died or " $N$ " for living. 


\section{Appendix D}

\section{Nurse Questionnaire: Post Implementation}

Were educational sessions helpful in regard to implementing the Care and Communication bundle? [YES/NO]

Were educational sessions helpful in regard to understanding the importance of implementing the Care and Communication Bundle? [YES/NO]

Did you find the Care and Communication Bundle easy to use? [YES/NO]

Did you find the Care and Communication Bundle to be effective in the intensive and cardiac care units? [YES/NO]

Is the Care and Communication Bundle, an order set that you believe that this facility could benefit from house wide? [YES/NO]

Comments: 